\title{
Run-out of cut-slope landslides: mesh-free simulations
}

\author{
X. $\mathrm{HE}^{*} \dagger$, D. LIANG $\dagger$ and M. D. BOLTON§
}

\begin{abstract}
This study uses an incompressible smoothed-particle hydrodynamics (ISPH) model to investigate the run-out and deposit morphology of granular materials flowing down cut slopes. The primary aim is to study the influence of various factors on the run-out and to summarise a quantitative relationship for direct use in landslide hazard management. In the model, the granular materials are modelled as a rigid perfectly plastic material with a Coulomb yield surface. The coupled continuity equation and momentum equation are solved by a semi-implicit algorithm. The model is first validated and its results are carefully compared with various controlled experiments regarding granular flows. The model reproduces the flows and correctly predicts the deposition profiles under various conditions. Then, the computational results are used to study the run-out and mobility of landslides. For granular columns collapsing onto a flat surface, a normalised run-out and a new scaling relationship are proposed, which are supported by numerous measured and numerical results. A similar relationship for the run-out of granular rectangles on steep slopes has also been explored. It is found that the normalised run-out is mainly determined by the slope angle and the normalised drop height. Furthermore, three types of idealised cut-slope landslides are simulated to study the influence of the initial landslide shape on the run-out. It is found that the normalised run-out of these idealised cut-slope landslides is smaller than that of granular rectangles on slopes of the same angles and drop heights. The difference between the run-outs is found to be mainly determined by the proportion of the whole mass that initially lies above a predictable discontinuity plane.
\end{abstract}

KEYWORDS: landslides; numerical modelling; slopes

\section{INTRODUCTION}

Owing to population growth, mountainous areas have become increasingly crowded. To make space for new infrastructure and to improve the environment, natural slopes are often reshaped into cut slopes. Some of these cut slopes are prone to landslides, which may cause fatalities and economic losses. To make proper use of land near cut slopes, it is necessary to assess the risk of cut-slope landslides and the subsequent run-out.

Early studies of the run-out of landslides relied largely on empirical formulae from statistical analyses of historical data. In these statistical models, the mobility of landslides is expressed by a well-known index called the reach angle. This index is defined as the angle of the line connecting the head of the landslide source to the distal margin of the deposited mass. This index was found to depend on the volume of the landslide mass (Corominas, 1996; Legros, 2002). In addition, Hunter \& Fell (2003) concluded that empirical models should also take into account dilatancy during the failure process, the geometry of the slope at and below the slide source area, and the degree of confinement of the travel path.

With the development of computing techniques and mathematical models, more and more physically based

Manuscript received 22 August 2016; revised manuscript accepted 12 April 2017.

Discussion on this paper is welcomed by the editor.

* Department of Engineering, University of Cambridge, Cambridge, UK (Orcid:0000-0001-5336-3663).

$\dagger$ Institut für Geotechnik, Universität für Bodenkultur, Wien, Austria (Orcid:0000-0001-5336-3663).

* Department of Engineering, University of Cambridge, Cambridge, UK (Orcid:0000-0001-5639-7375).

$\S$ Department of Engineering, University of Cambridge, Cambridge, UK. dynamic methods have emerged. A widely used continuumbased dynamic model was constructed by Savage \& Hutter (1989). They incorporated the Mohr-Coulomb model into a set of depth-averaged equations for dry granular flows. Iverson \& Denlinger (2001) then extended these depth-averaged equations to two-phase flows based on mixture theory.

The smoothed-particle hydrodynamics (SPH) numerical method has increasingly been used in continuum-based modelling. The SPH method was initially applied in astrophysics and fluid dynamics. Efforts have also been made to implement the SPH method in soil mechanics models to study slope stability (Bui et al., 2011) and large deformations of soils (Bui et al., 2008). It was recently used to simulate the collapse of granular columns (Liang \& He, 2014; He \& Liang, 2015). However, the fully dynamic SPH method has not been used for studying cut-slope landslide phenomena, which involve violent impact and complex topography.

This paper reports on a numerical investigation of the run-out and deposit morphology of cut-slope landslides. Unlike previous SPH models in the geotechnical community (Bui et al., 2011; An et al., 2016; Nguyen et al., 2016), which update the mean stress from an explicit algebraic equation, this study adopts an SPH method, which solves the mean stress implicitly. The mathematical model is first verified against experimental results of dry granular flows in various configurations. It is shown that the present SPH model can faithfully predict the flows, even when violent impact happens. Consistent with the findings in computational fluid dynamics, the present implicit SPH model demonstrates advantages over the explicit SPH models by obtaining a smooth stress field without special stabilisation techniques and allowing a greater time step. The primary focus of this study is the run-out of landslides. First, the extensively discussed scaling relationship for the run-out of granular columns is re-examined. A new normalised run-out is 
proposed to better indicate the relative mobility. Correspondingly, a new normalised relationship between this normalised run-out and initial geometrical parameters is suggested, which is supported by numerous experimental results and past and present numerical simulations. Then, a similar normalised relationship for the run-out of granular rectangles released on cut slopes is explored for the first time. Finally, the SPH model is applied to simulate some idealised cut-slope landslides, which are a reasonable abstraction of real-world landslides.

\section{MATHEMATICAL MODEL}

In the present study, the flow-type landslide mass is modelled as an incompressible material as in Balmforth \& Kerswell (2005) and Ionescu et al. (2015) with the governing equations as follows.

$$
\begin{aligned}
& \frac{\mathrm{d} \rho}{\mathrm{d} t}=-\rho \frac{\partial \boldsymbol{v}_{i}}{\partial x_{i}}=0 \\
& \frac{\mathrm{d} v_{i}}{\mathrm{~d} t}=-\frac{1}{\rho} \frac{\partial \boldsymbol{\sigma}_{i j}}{\partial x_{j}}+\boldsymbol{g}_{i}=-\frac{1}{\rho} \frac{\partial p}{\partial x_{i}}-\frac{1}{\rho} \frac{\partial s_{i j}}{\partial x_{j}}+\boldsymbol{g}_{i}
\end{aligned}
$$

Here, $\rho=\left[(1+w) \rho_{\text {solid }}\right] /(1+e)$ is the bulk density of the soil, with $\rho_{\text {solid }}, w$ and $e$ the density of the solid, moisture content and void ratio, respectively. During the rapid flowing of the soil mass, the density of solid, the void ratio and the moisture content are assumed to be constants to meet the incompressible condition. $\boldsymbol{\sigma}_{i j}=p \boldsymbol{\delta}_{i j}+\boldsymbol{s}_{i j}$ is the stress tensor. $p$ is the mean stress. $\boldsymbol{s}_{i j}$ is the deviatoric stress tensor. $\boldsymbol{g}_{i}$ is the acceleration due to gravity. This paper adopts the convention commonly used in soil mechanics that compressive stress and strain are positive.

\section{ISPH scheme}

Using the authors' in-house computer code, the coupled continuity and momentum equations are solved by an incompressible smoothed-particle hydrodynamics (ISPH) method. Readers are referred to He (2016) for the architecture of the computer program and the detailed numerical treatments. The incompressibility condition is imposed by a projection method comprising a predictor step and a corrector step. In the predictor step, an intermediate velocity field is obtained without satisfying the continuity condition. The errors in the predicted velocity field are then corrected by solving a pressure Poisson equation (Shao, 2012).

The present study chooses the quintic Wendland smoothing kernel $W(\boldsymbol{x}, h)$, where $h$ denotes the smoothing length and $\boldsymbol{x}$ denotes the position vector. For two neighbouring particles at positions $\boldsymbol{x}^{a}$ and $\boldsymbol{x}^{b}$, respectively, the short notation $W^{a b}=W\left(x^{a}-x^{b}, h\right)$ and $\nabla_{j} W^{a b}=\left(\partial W / \partial x_{j}\right)\left(\boldsymbol{x}^{a}-\boldsymbol{x}^{b}, h\right)$ is used. Symbols $f^{a, n}$ and $f^{a, n+1}$ denote the values of a function at particle $a$ at time $t^{n}$ and time $t^{n+1}=t^{n}+\Delta t$, respectively. If the time step index is omitted in an equation, then the values are all evaluated at the same time.

The predictor step is the integration of the momentum equation in time considering only the gravity term and deviatoric stress term. The intermediate velocity is calculated as

$$
\frac{\boldsymbol{v}_{i}^{a, n *}-\boldsymbol{v}_{i}^{a, n}}{\Delta t}=-\sum_{b}^{N_{\text {nei }}} m^{b}\left[\frac{\boldsymbol{s}_{i j}^{a, n}}{\left(\rho^{a}\right)^{2}}+\frac{\boldsymbol{s}_{i j}^{b, n}}{\left(\rho^{b}\right)^{2}}\right] \nabla_{j} W^{a b, n}+\boldsymbol{g}_{i}
$$

Here, $f^{n^{*}}$ denotes the intermediate value when marching from $t^{n}$ to $t^{n+1}$. In the predictor step, the continuity equation is not considered. Then a corrector step is used to adjust the intermediate velocity by taking into account the pressure gradient term.

$$
\frac{\boldsymbol{v}_{i}^{a, n+1}-\boldsymbol{v}_{i}^{a, n *}}{\Delta t}=-\sum_{b}^{N_{\text {nei }}} m^{b}\left[\frac{p^{a, n+1}}{\left(\rho^{a}\right)^{2}}+\frac{p^{b, n+1}}{\left(\rho^{b}\right)^{2}}\right] \nabla_{j} W^{a b, n}
$$

In equations (3) and (4), the SPH formations are used to estimate the gradient of the mean stress and deviatoric stress. The following Poisson equation should be used to calculate the pressure field, which will generate the correct pressure gradient term in equation (4) to ensure the conservation of mass at $t^{n+1}$.

$$
\begin{aligned}
{\left[\frac{\partial}{\partial x_{j}}\left(\frac{1}{\rho} \frac{\partial p}{\partial x_{j}}\right)\right]^{a, n+1} } & =\sum_{b}^{N_{\text {nei }}} m^{b} \frac{8}{\left(\rho^{a}+\rho^{b}\right)^{2}} \frac{p^{a, n+1}-p^{b, n+1}}{\left(r^{a b, n}\right)^{2}+\eta^{2}} x_{j}^{a b} \nabla_{j} W^{a b, n} \\
& =\frac{\rho^{a, n}-\rho^{a, n *}}{\rho^{a, n} \Delta t^{2}}
\end{aligned}
$$

Here, $\eta$ is a small value, taken to be $0 \cdot 1 h$, to keep the denominator non-zero even if two particles are very close. In equation (5), the intermediate density is approximated to be $\rho^{a, n *}=\sum_{b}^{N_{\text {nei }}} m^{b} W^{a b *}$, whereas the initial density is estimated to be $\rho^{a, n}=\sum_{b}^{N_{\text {nei }}} \mathrm{m}^{b} W^{a b, n}$.

In this ISPH method, there is no need for the introduction of any sound speed. Hence, the Courant criterion is based on the particle velocities rather than the wave speed, which allows a bigger time step than other explicit SPH models (Liang, 2010; Bui et al., 2011; An et al., 2016).

$$
\Delta t \leq C_{\max } \frac{\Delta l}{\left|\boldsymbol{v}_{i}\right|_{\max }}
$$

Here, $\left|\boldsymbol{v}_{i}\right|_{\max }$ is the maximum velocity and $\Delta l$ is the initial spacing of particles.

\section{The Mohr-Coulomb model}

There have been extensive research studies on the mechanics of granular materials. During the mobilisation stage, the evolution of the frictional resistance and dilatancy depends on the initial state of the soil (Bolton, 1986). After a so-called critical state is reached after large deformations, however, the coefficient of friction resistance remains constant and the dilatancy becomes zero (Schofield \& Wroth, 1968). Because the soil elements in flow-type landslides will already have suffered large deformations during the landslide triggering phase, they should stay in a critical state while they continue to deform. In this study, the soil is modelled as a rigid-perfectly plastic material with a Coulomb yield surface (Fig. 1). On the failure plane ( $\mathrm{F}$ in Fig. 1), the shear stress $\tau$ and the effective normal stress $\sigma^{\prime}$ have the following relationship.

$$
\tau=\sigma^{\prime} \tan \phi_{\text {crit }}
$$

where $\phi_{\text {crit }}$ is the internal friction angle, chosen as the critical state value to model flow-type landslides. In plane-strain problems, the deviatoric stress is $\left|\boldsymbol{s}_{i j}\right|=\left(\sigma_{1}^{\prime}-\sigma_{3}^{\prime}\right) / 2$ and the mean stress is $p=\left(\sigma_{1}^{\prime}+\sigma_{3}^{\prime}\right) / 2$. Here, $\sigma_{1}^{\prime}$ and $\sigma_{3}^{\prime}$ are the effective principal stresses. In this paper, the norm of a second-order tensor is defined as the square root of the second deviatoric invariant of that tensor. It is easy to verify that $\left|\boldsymbol{s}_{i j}\right|=p \sin \phi_{\text {crit }}$. Studies show that the stress tensor and strain rate tensor are coaxial at the critical state in simple shear (Ai et al., 2014) and transient flow (Lacaze \& Kerswell, 2009) conditions. With such a coaxial assumption, the following relationship is implemented in SPH. 


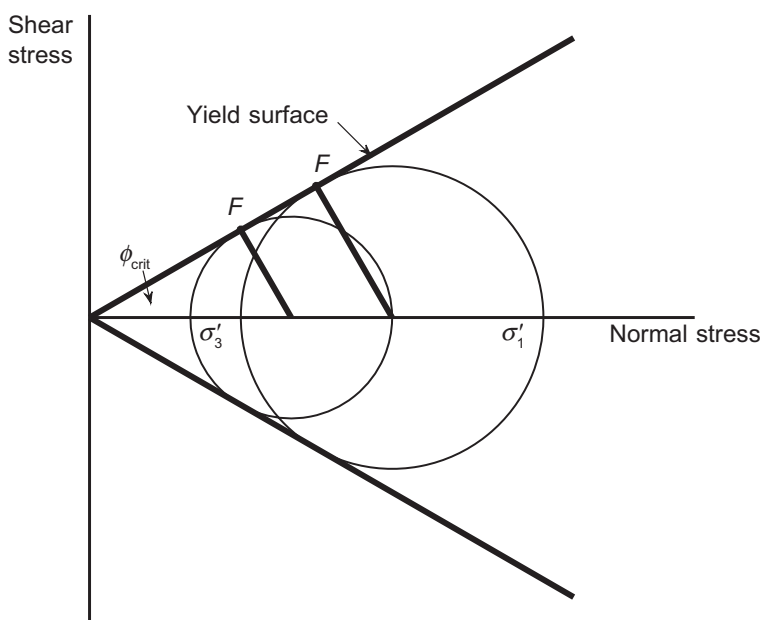

Fig. 1. Mohr diagram showing the Coulomb yield surface

$$
\boldsymbol{s}_{i j}=p \sin \phi_{\text {crit }} \frac{\dot{\boldsymbol{\epsilon}}_{i j}}{\left|\dot{\boldsymbol{\epsilon}}_{i j}\right|}
$$

Here, $\dot{\epsilon}_{i j}$ is the deviatoric strain rate tensor. The relationship described by this equation is often referred to as the DruckerPrager yield criterion (Schofield \& Wroth, 1968).

The accuracy of the SPH approximation is severely reduced near boundaries due to particle deficiency. In this study, the corrected gradient of the kernel is used for the estimation of strain rate to improve the accuracy, as expressed below

$$
\left(\dot{\boldsymbol{\varepsilon}}_{i j}\right)^{a}=\frac{1}{2} \sum_{b}^{N_{\text {nei }}} \frac{m^{b}}{\rho^{b}}\left[\left(\boldsymbol{v}_{i}^{a}-\boldsymbol{v}_{i}^{b}\right) \tilde{\nabla}_{j} W^{a b}+\left(v_{j}^{a}-v_{j}^{b}\right) \tilde{\nabla}_{i} W^{a b}\right]
$$

Here, $\tilde{\nabla_{j}} W^{a b}=\boldsymbol{B}_{i j}^{-2, a} \nabla_{i} W^{a b}$ and $\boldsymbol{B}_{i j}^{-2, a}$ is the inverse of $\boldsymbol{B}_{i j}^{2, a}=\sum_{b}^{N_{\text {nei }}}\left(m^{b} / \rho^{b}\right)\left(x_{i}^{b}-x_{i}^{a}\right) \nabla_{j} W^{a b} . \tilde{\nabla}_{j} W^{a b}$ is termed as the renormalised gradient of the kernel (Randles \& Libersky, 1996).

In summary, the soil model is implemented in the predictor step. The strain rate tensor for every particle is first estimated using equation (9). For incompressible materials, the strain rate tensor should be equal to the deviatoric strain rate tensor. However, the strain rate tensor calculated from equation (9) is often not exactly a deviatoric tensor due to numerical errors. The deviatoric strain rate tensor is still evaluated as $\dot{\boldsymbol{\epsilon}}_{i j}=\dot{\boldsymbol{\varepsilon}}_{i j}-\left(\dot{\boldsymbol{\varepsilon}}_{\mathrm{kk}} / 2\right) \boldsymbol{\delta}_{i j}$ and the deviatoric stress tensor is then updated using equation (8).

\section{Boundary treatments}

For particles near or on the boundary, only the neighbouring particles inside the domain contribute to the SPH approximation, and no contribution comes beyond the boundary. This one-sided contribution leads to inaccuracy, but this can be mitigated by taking additional steps to preserve the conservation of mass and momentum at the boundaries.

The free surface condition is implemented on a set of the most outlying particles. Owing to particle deficiency in the evaluation of the gradient of stresses in equations (3) and (4), particles near the free surface tend to cluster together during simulations. In this paper, the mean stress of free surface particles is assigned a small value of $\rho g \Delta l / 4$. This will shift the mean stress field by this value, but will have no influence on the estimation of the gradient of stresses for interior particles.

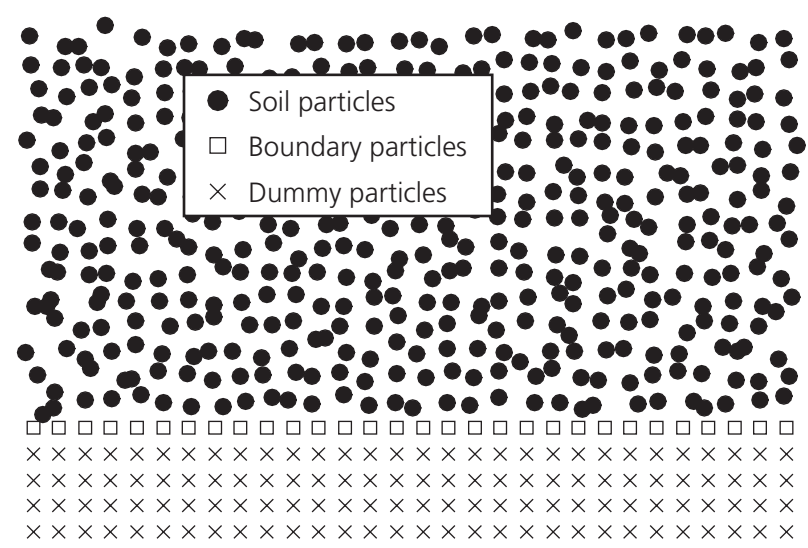

Fig. 2. Boundary particles and dummy particles for the treatment of fixed boundary

This treatment will compensate for the missing contributions for particles near the free surface and avoid the cluster of particles. The free surface particles are easily tracked as their SPH estimation of density drops abruptly due to particle deficiency. The criterion used for the detection of free surface particles is $\rho^{a, n}<0 \cdot 97 \rho^{0}$.

At the fixed boundary, a non-penetration Coulomb friction condition is enforced. In this study, a combination of boundary particles and dummy particles is used to model the fixed boundary (Fig. 2). In this technique, one layer of fixed particles is placed on the boundary and several layers of dummy particles are placed outside the boundary. During calculations, the velocity and position of boundary particles and dummy particles are not updated. However, the mean stress of boundary particles is calculated through the pressure Poisson equation and the deviatoric stress is updated through equations similar to equation (8). As for the stress of dummy particles, a uniform distribution of stress is assumed on and outside the fixed boundary. For a dummy particle $d$, it has an associated boundary particle $b(d)$ which is the closest to it among all boundary particles. The stress on this dummy particle is specified to be the same as the stress on its associated boundary particle. Such a treatment helps to achieve the Neumann boundary condition $\left(\left.\left(\partial / \partial x_{i}\right) \boldsymbol{n}_{\boldsymbol{i}}\right|_{\partial \mathrm{w}} \Omega=0\right)$ when solving the Poisson equation, as the position vector $\boldsymbol{x}_{j}^{b(d)}-\boldsymbol{x}_{j}^{d}$ between a dummy particle and its associated boundary particle is approximately in the normal direction $\left(\boldsymbol{x}_{j}^{b(d)}-\boldsymbol{x}_{j}^{d} \approx \pm\left|\boldsymbol{x}_{j}^{b(d)}-\boldsymbol{x}_{j}^{d}\right| \boldsymbol{n}_{j}\right)$. Here, $\boldsymbol{n}_{j}$ is the unit vector normal to the fixed boundary. In this way, the pressure at boundary particles is able to repel the approaching soil particles to avoid particle penetration.

\section{COLLAPSE OF GRANULAR COLUMNS ONTO HORIZONTAL AND INCLINED PLANES}

Most of the studies of landslides are restricted to the movement of the front and the shape of the deposition. The understanding of the flow and deposition mechanisms remains qualitative. Recently, a number of small-scale laboratory experiments of granular materials collapsing onto horizontal and inclined planes have been reported (Balmforth \& Kerswell, 2005; Lajeunesse et al., 2005; Lube et al., 2005, 2011; Lacaze et al., 2008). These phenomena have also been simulated with discrete-element model (DEM) (Staron \& Hinch, 2005; Lacaze et al., 2008; Utili et al., 2015) and continuum-based numerical methods (Crosta et al., 2009). These granular collapse phenomena are 


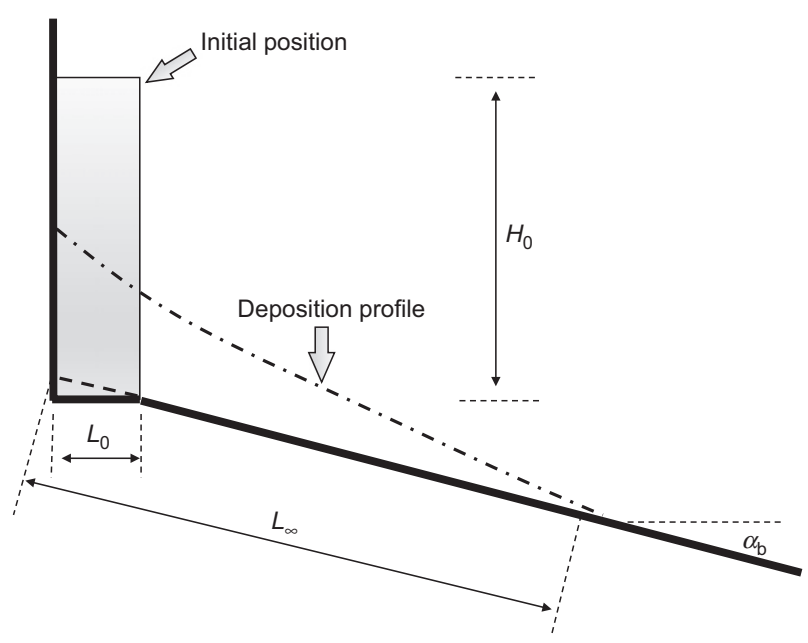

Fig. 3. Set-up of the collapse of granular columns

well defined and share some similarities with landslides, providing an opportunity to assess various dynamic models. The set-up of the problem is illustrated in Fig. 3. Granular columns are initially contained in a rectangular box. The initial width and height of the columns are denoted as $L_{0}$ and $H_{0}$, respectively. The aspect ratio is $a=H_{0} / L_{0}$. There is a removable gate on one side of the box, which is connected to a horizontal or inclined plane. Once the gate is suddenly removed, the granular column will collapse onto the plane, before finally stopping to form a heap. The run-out is defined as the length of the deposition along the plane.

\section{Demonstration of the collapsing process}

Nguyen et al. (2016) conducted a series of twodimensional (2D) collapse experiment onto a horizontal plane with aluminium rods $50 \mathrm{~mm}$ long. The diameters of the rods were 1.6 or $3.0 \mathrm{~mm}$, and they were mixed in the ratio of $5 \cdot 27: 1$ by number, which resulted in a bulk density of $2079 \cdot 5 \mathrm{~kg} / \mathrm{m}^{3}$. The internal friction angle was measured to be $21.9^{\circ}$ in biaxial tests (Nguyen et al., 2016). This value is assumed to be the critical state friction angle and is employed in the present simulation. Fig. 4 presents the SPH-simulated collapse of a shallow column $\left(L_{0}=0.2 \mathrm{~m}, H_{0}=0.1 \mathrm{~m}\right)$. In comparison with experiments, the time evolution of the free surface, especially the deposition profile, is predicted fairly well by the ISPH model.

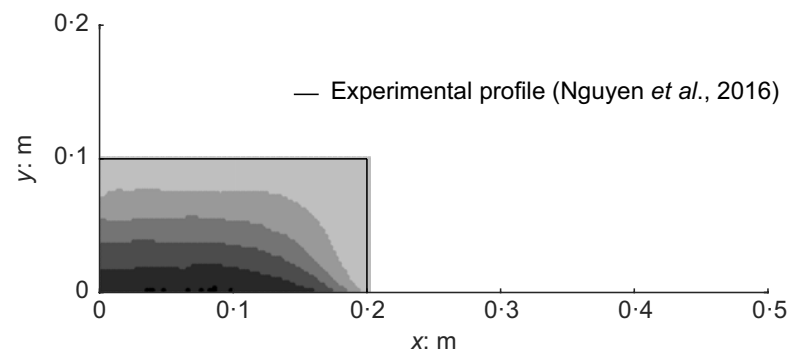

(a)

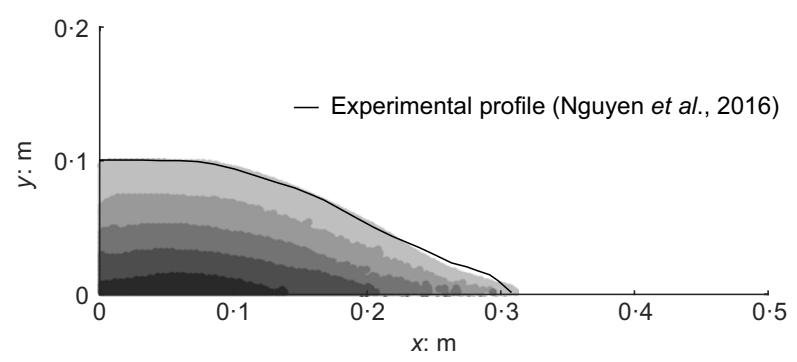

(c)

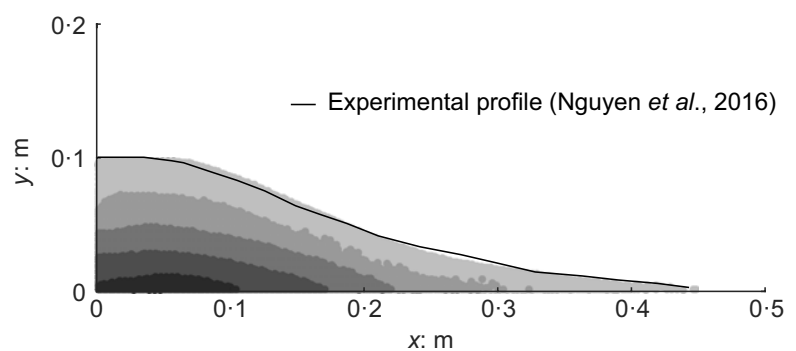

(e)

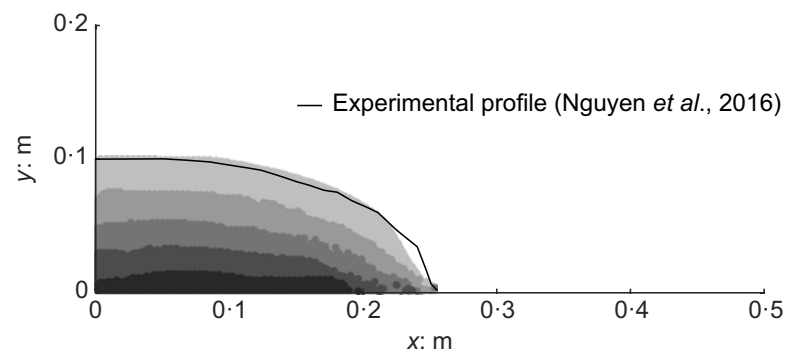

(b)

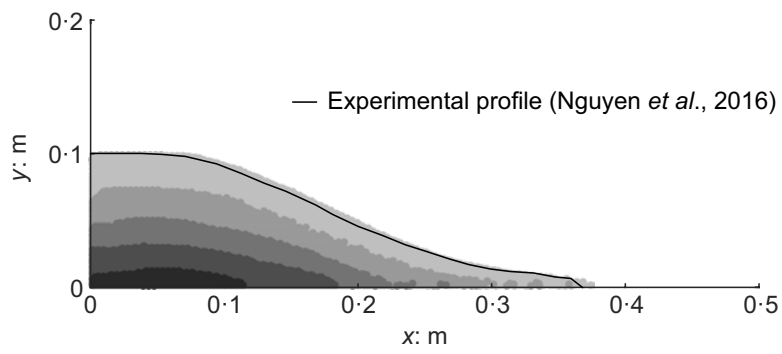

(d)

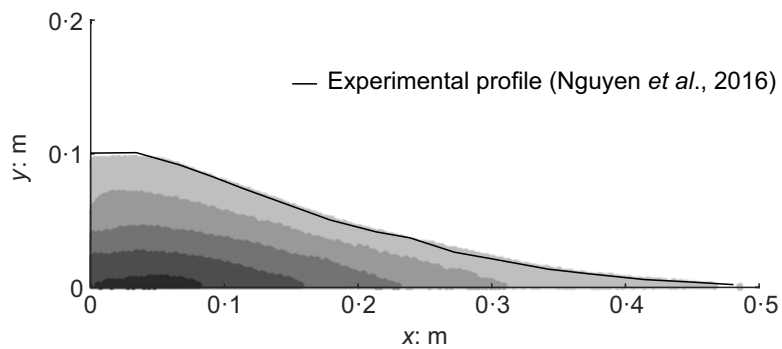

(f)

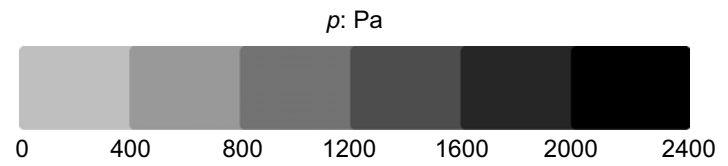

Fig. 4. Collapsing processes of a shallow column on a horizontal plane $\left(L_{0}=0 \cdot 2 \mathrm{~m}, H_{0}=0 \cdot 1 \mathrm{~m}\right)$ : (a) $t=0 \cdot 005 \mathrm{~s} ;(\mathrm{b}) t=0 \cdot 109 \mathrm{~s} ;$ (c) $t=0 \cdot 172 \mathrm{~s}$; (d) $t=0.235 \mathrm{~s}$; (e) $t=0.335 \mathrm{~s}$; (f) $t=0.607 \mathrm{~s}$ 
It is generally recognised in the literature that those numerical models that update the mean stress from explicit equations suffer from unphysical stress/pressure oscillations and this is also true for SPH models (Bui et al., 2011; Nguyen et al., 2016). Some stabilisation techniques have been developed to remove the oscillations successfully, which include the introduction of an artificial viscosity (Monaghan, 1992; Bui et al., 2011), stress/strain regularisations (Nguyen et al., 2016) and so on. The implicit SPH models have also been proved in many hydrodynamic applications to be able to successfully reduce the oscillations (Lee et al., 2008; Shao, 2012). In the current simulations, the mean stress distribution is monitored during the collapse, as plotted in Fig. 4. It is observed that, without any sophisticated stabilisation technique, a relatively smooth stress field is obtained with the present ISPH model. To the best of the authors' knowledge, the present study is the first application of the ISPH model to geotechnical problems.

\section{Deposition of slowly poured granular materials}

When bulk granular materials are poured slowly onto a horizontal surface, a heap will form. The angle between the top surface of the heap and the horizontal plane is known as the angle of repose (AOR), which is a commonly used parameter for measuring the friction of granular materials. This phenomenon is simulated with the present ISPH model, with a procedure illustrated in Fig. 5. The initial spacing of particles is $\Delta l=0.01 \mathrm{~m}$. A tall column with $L_{0}=20 \Delta l$ and $H_{0}=30 \Delta l$ is first released. Then, several shallow, granular columns, $L_{\text {rel }}$ wide and $H_{\text {rel }}$ high, are released just above the deposition multiple times, successively. $L_{\mathrm{rel}}$ is fixed at $15 \Delta l$, and four series of simulations are conducted with $H_{\text {rel }}$ of $\Delta l, 5 \Delta l, 10 \Delta l$ or $15 \Delta l$, respectively. $\phi_{\text {crit }}$ is $36 \cdot 5^{\circ}$ throughout the simulations.

The successive release of shallow columns just above the deposition ensures that the level of flow inertia is low, which

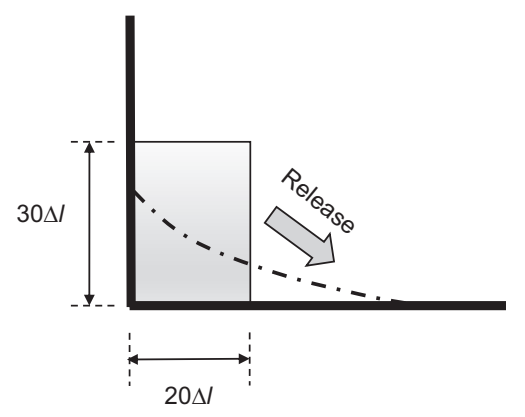

(a)

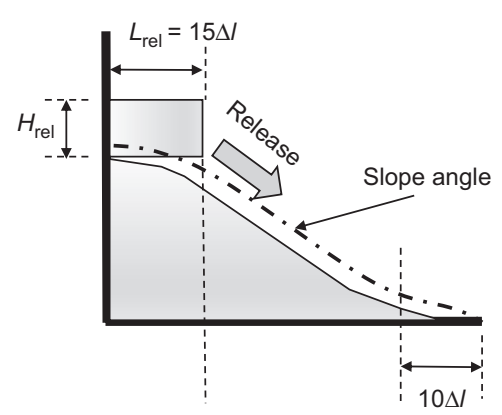

(b)

Fig. 5. SPH simulation of a heap formed by slowly pouring granular materials: (a) initial release of a tall, granular column; (b) subsequent release of a series of shallow, granular columns on top of the existing deposition

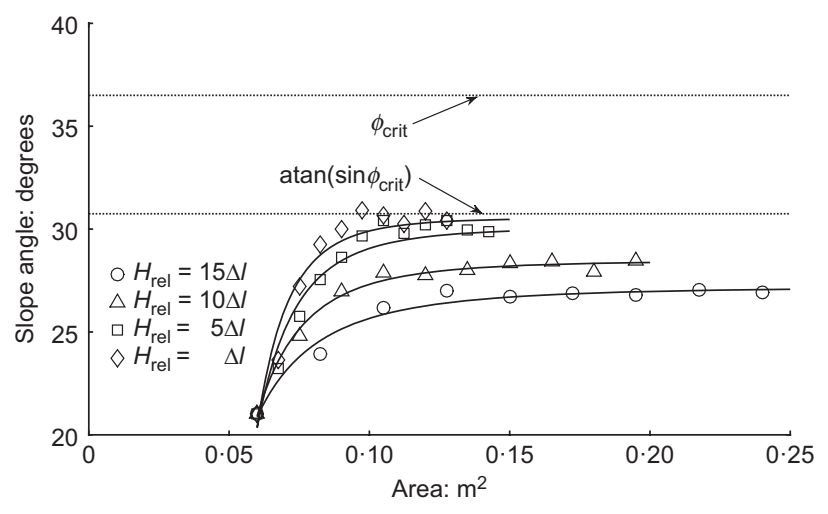

Fig. 6. Relationships between the slope angle and the heap crosssectional area for the release of different granular columns on the top

mimics the 'slow pouring' of granular materials. The slope angle in the middle part of the deposition (Fig. 5(b)) is measured, because the head and the toe are greatly affected by the boundaries. The variation of the slope angle is plotted in Fig. 6. When a tall column is first released, the slope angle is about $21^{\circ}$, which deviates greatly from $\phi_{\text {crit }}$ due to inertial effects. However, when granular material is successively poured over the deposition, the slope angle increases. An asymptotic slope angle is achieved after many such depositions. Fig. 6 also shows that the asymptotic slope angle increases as the height $H_{\text {rel }}$ reduces, which also reduces inertial effects during deposition. When $H_{\text {rel }}$ reduces from $5 \Delta l$ to $\Delta l$, the asymptotic slope angle does not observe a significant increase. Hence, it may be assumed that inertial effects have become insignificant. If the asymptotic slope angle of the deposition when inertia effects are negligible is regarded as the AOR, then the AOR appears to be somewhat smaller than the critical friction angle. Fig. 6 suggests a general specification of $\tan \mathrm{AOR} \approx \sin \phi_{\text {crit }}$, which happens to be consistent with the hypothesis of Roscoe (1970) for the friction angle mobilised on slip lines of zero extension.

\section{Deposition profiles}

It has been verified that all the key features of the collapse observed in experiments can be faithfully reproduced with the present ISPH model (Liang \& He, 2014; He \& Liang, 2015). Its performance in predicting the final deposition profiles in various configurations is summarised in Fig. 7. The profiles from experiments are plotted with solid lines and the SPH results are labelled with dashed lines. Fig. 7(a) shows the comparison of the deposition profiles between the experiments and SPH simulations at various values of $\phi_{\text {crit }}$. The experimentally measured deposition profile is better reproduced by the ISPH model when choosing $\sin \phi_{\text {crit }}=\tan$ AOR, which is consistent with argument in the previous section. Fig. 7(b) shows the deposition profiles of the grit columns with different initial geometry. Columns with a greater cross-sectional area $S_{0}=H_{0} L_{0}$ (column A) produce a greater run-out. When two columns with the same $S_{0}$ (columns B and C) are prepared, the column with a higher aspect ratio (column C) travels further. Fig. 7(c) compares the collapse of columns of the same initial geometry $\left(L_{0}=2 \mathrm{~cm}\right.$, $H_{0}=25 \mathrm{~cm}$ ) but with different material compositions. It is shown that the column of materials with a smaller AOR (the glass balls) travels further. Fig. 7(d) compares the deposition of the same columns onto different slopes. It is observed that material travels further down on steeper slopes. The good agreement with the measurements shows that the present 


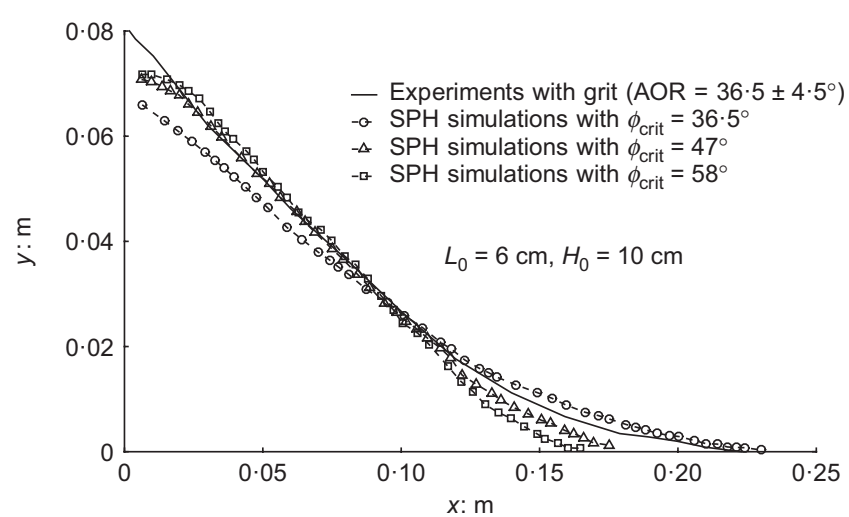

(a)

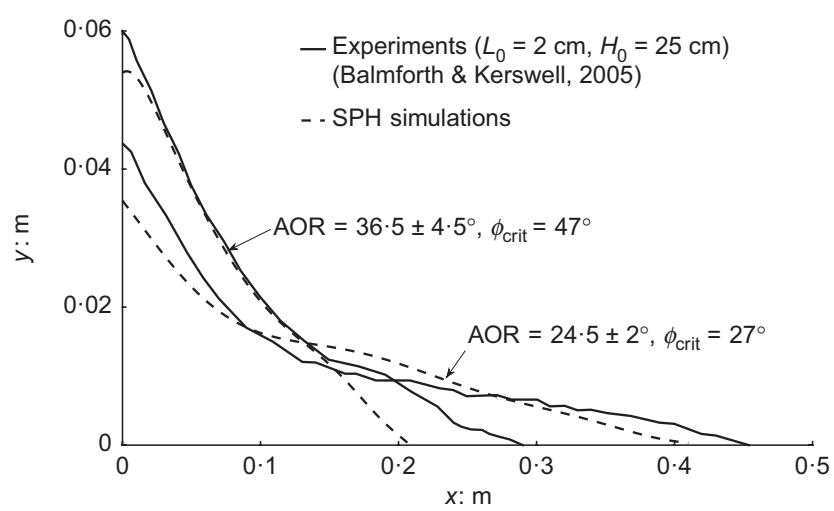

(c)

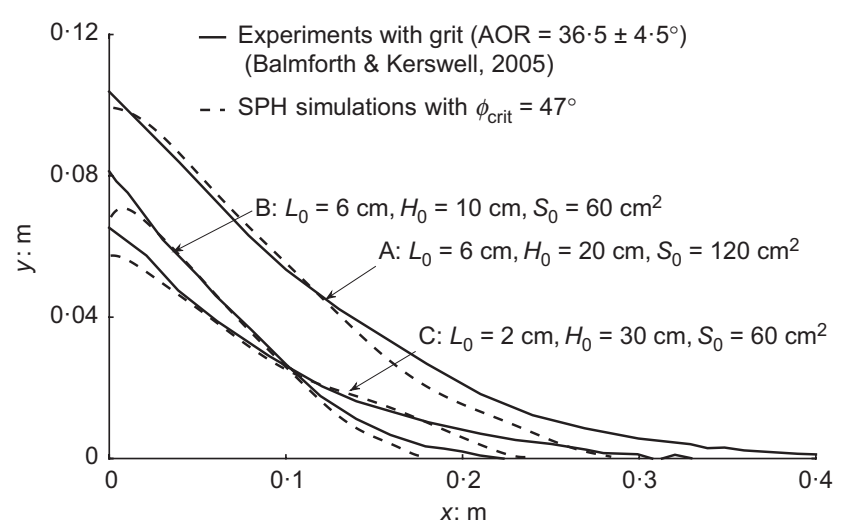

(b)

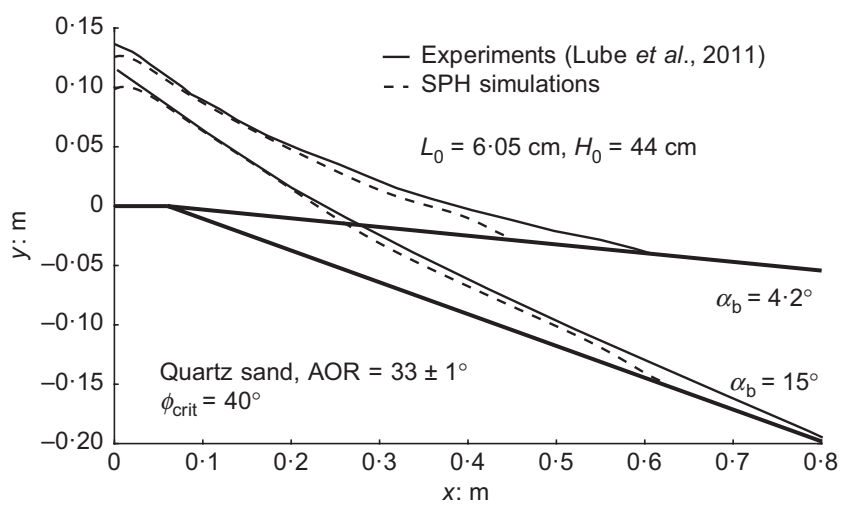

(d)

Fig. 7. Deposition profile of tall columns: (a) SPH-simulated deposition profiles with various critical state friction angles; (b) deposition profiles of columns with different initial dimensions; (c) deposition profiles of columns with different materials; (d) deposition profiles onto slopes of different angles

Table 1. Correspondence between AOR and $\phi_{\text {crit }}$

\begin{tabular}{|c|c|c|c|}
\hline Source & $\phi_{\text {crit }}$ used in simulations & $\operatorname{atan}\left(\sin \phi_{\text {crit }}\right)$ & AOR observed in experiments \\
\hline $\begin{array}{l}\text { Figure } 6 \\
\text { Numerical simulation }\end{array}$ & $36 \cdot 5^{\circ}$ & $30 \cdot 7^{\circ}$ & $30 \cdot 5^{\circ}$ \\
\hline $\begin{array}{l}\text { Figures } 7(a) \text { and } 7(b) \\
\text { Experiment }- \text { grit }\end{array}$ & $47 \cdot 0^{\circ}$ & $36 \cdot 2^{\circ}$ & $36 \cdot 5^{\circ} \pm 4 \cdot 5^{\circ}$ \\
\hline $\begin{array}{l}\text { Figure } 7(\mathrm{c}) \\
\quad \text { Experiment - glass balls }\end{array}$ & $27 \cdot 0^{\circ}$ & $24 \cdot 4^{\circ}$ & $24 \cdot 5^{\circ} \pm 2 \cdot 0^{\circ}$ \\
\hline $\begin{array}{l}\text { Figure } 7(\mathrm{~d}) \\
\text { Experiment - quartz sand }\end{array}$ & $40 \cdot 0^{\circ}$ & $32 \cdot 7^{\circ}$ & $33 \cdot 0^{\circ} \pm 1 \cdot 0^{\circ}$ \\
\hline
\end{tabular}

model can correctly capture the mobility features of granular columns in various conditions. Table 1 compares the relationship between $\phi_{\text {crit }}$ and AOR, as used in the simulations and quoted in the experiments, which supports the proposition $\tan \mathrm{AOR} \approx \sin \phi_{\text {crit }}$.

The small discrepancies between the measured and computed results can be attributed to the limitations of the present model. First, the present ISPH model cannot consider the volume or density change of the collapse. However, granular materials usually contract or dilate, depending on their initial state, to a critical state void ratio during the mobilisation process. The void ratio in a flowing state has also been reported to be related to the local mean stress and shear rate and thus is not a constant (Forterre \& Pouliquen, 2008). The neglect of these features leads to a slight deviation in the final cross-sectional area. The ISPH model always maintains the same cross-sectional area $\left(S_{\infty} \approx S_{0}\right)$, whereas the experiments found that $S_{\infty}$ is approximately $10 \%$ larger than $S_{0}$ (Balmforth \& Kerswell, 2005), which must mainly be attributed to dilation in the early stages of mobilisation. Second, recent physical experiments confirm that the critical state friction coefficient is not constant, and is greater in an inertia regime than the conventional coefficient measured in a quasi-static regime (GDR MiDi, 2004). This finding is likely to explain the inaccurate prediction of the front of the flow, where the rate of shearing is high and the mean stress is low.

A key finding in the literature (Balmforth \& Kerswell, 2005; Lajeunesse et al., 2005; Lube et al., 2005; Staron \& Hinch, 2005) concerning the collapse of granular columns is a scaling relationship between the non-dimensional run-out $\left(\left(L_{\infty}-L_{0}\right) / L_{0}\right)$ and the initial aspect ratio $\left(a=H_{0} / L_{0}\right)$. When the column is shallow, they are found to have a linear relationship $\left(\left(L_{\infty}-L_{0}\right) / L_{0}=\lambda_{1} a\right)$. When the column is slender, a power relationship $\left(\left(L_{\infty}-L_{0}\right) / L_{0}=\lambda_{2} a^{\beta}\right)$ is suggested. 


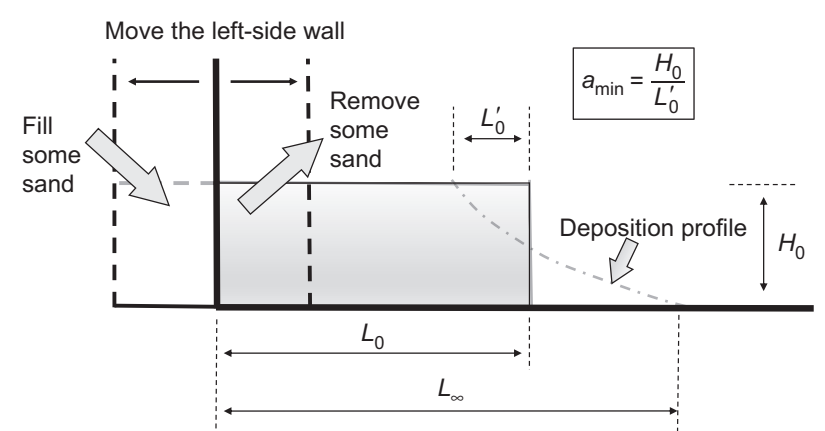

(a)

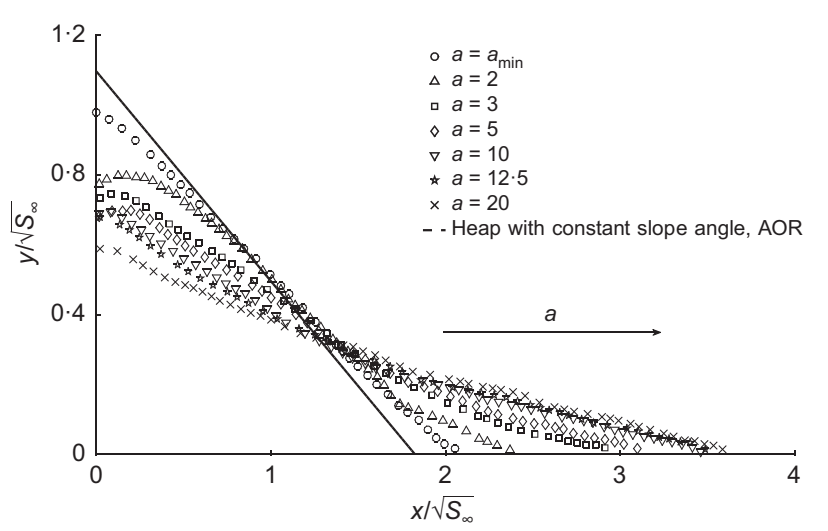

(b)

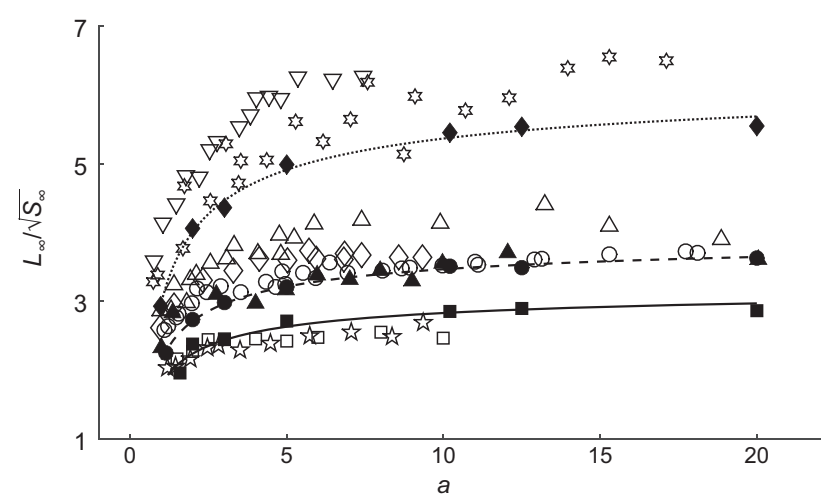

(c)

Fig. 8. Normalised deposition profiles and the normalised run-outs on flat beds: (a) an illustration of the shallow-column collapse; (b) SPH-predicted normalised deposition profiles; (c) relationships between the normalised run-out and the initial aspect ratio, with the three lines representing the proposed scaling law for results of this study

A new scaling law and the mobility of granular columns

In this section, the conventional scaling relationship is first reinterpreted and then another formulation is proposed to facilitate the analyses of landslides involving complex geometries.

The collapse processes of shallow columns of different aspect ratios are not fundamentally different, and the height of the column will be chosen as a characteristic length. As illustrated in Fig. 8(a), the solid black lines represent the left-side wall and the base. The shaded area is the initial shallow column to be collapsed onto the base to the right. Because the left part of the column remains static and does not participate in the flow at all, there should be no influence on the collapse if either $(a)$ the left-side wall is moved leftward and simultaneously the gap is filled with soil or $(b)$ some soil is removed and simultaneously the left-side wall is moved rightward. Despite the change of the aspect ratio $a=H_{0} / L_{0}$ in case $(a)$ and case $(b)$, the collapse process is fundamentally the same. This leads to a linear relationship between $\left(L_{\infty}-L_{0}\right) / L_{0}$ and $a$ for small $a$. From the deposition profiles of shallow columns, a minimum aspect ratio $a_{\min }$, as shown in Fig. 8(a), can be identified such that the deposition profiles of columns with aspect ratios smaller than $a_{\min }$ are all similar.

However, when the column is high enough, it is found in the literature that the growth of $\left(L_{\infty}-L_{0}\right) / L_{0}$ with $a=H_{0} / L_{0}$ follows a power function $\left(\left(L_{\infty}-L_{0}\right) / L_{0}=\lambda_{2} a^{\beta}\right.$, with $\beta=2 / 3$ in Lajeunesse et al. (2005) and Lube et al. (2005)). However, the growth of $\left(L_{\infty}-L_{0}\right) / L_{0}$ with $a$ is partly attributed to the mass increase of granular material contributing to the flow. When the level of mobility is compared in a generic sense, non-dimensional parameters should be used to get rid of the effect of the column volume or mass. For example, considering $(a)$ a statically deposited heap with large volume and (b) a very high column with small volume, it is possible that the statically deposited heap has a greater absolute run-out than the high column. Nevertheless, the tendency is to regard the high column as more mobile. Therefore, a new dimensionless run-out is needed, which can reflect such relative mobility.

Figure 8(b) shows the deposition profiles normalised by $\sqrt{S_{\infty}}$ for ISPH simulations with various aspect ratios. Here, $S_{\infty}$ is the cross-sectional area of the final heap. For columns of the same material and the same aspect ratio (whereas $L_{0}$, $H_{0}$ and $S_{0}$ may be different), the normalised profiles are seen to collapse together. All these normalised profiles have a unity cross-sectional area. When the aspect ratio is equal to $a_{\text {min }}$, the deposition profile is the closest to a statically deposited heap with a slope angle slightly smaller than the AOR. When the aspect ratio increases, the granular column becomes more mobile, the normalised run-out $L_{\infty} / \sqrt{S_{\infty}}$ will increase and the normalised profile spreads outwards.

The figure shows that the normalised run-out $L_{\infty} / \sqrt{S_{\infty}}$ depends only on the initial dimensionless geometrical parameter, namely, the aspect ratio, for a given granular material. Here, $L_{\infty} / \sqrt{S_{\infty}}$ is the preferred dimensionless run-out because it properly quantifies the relative mobility of the statically deposited heap and the slender column as discussed above. Because the cross-sectional area during most of the collapse process is close to that of the final deposition in experiments, $\sqrt{S_{\infty}}$ is chosen as the characteristic length. This has no effect on the SPH results, where the cross-sectional area is assumed constant.

The relationship between $L_{\infty} / \sqrt{S_{\infty}}$ and $a$ is presented in Fig. 8(c). This figure includes data from laboratory experiments, DEM simulations and continuum-based computations. Data from experiments and DEM simulations are presented with $L_{\infty} / \sqrt{S_{0}}$ due to the lack of dilation 


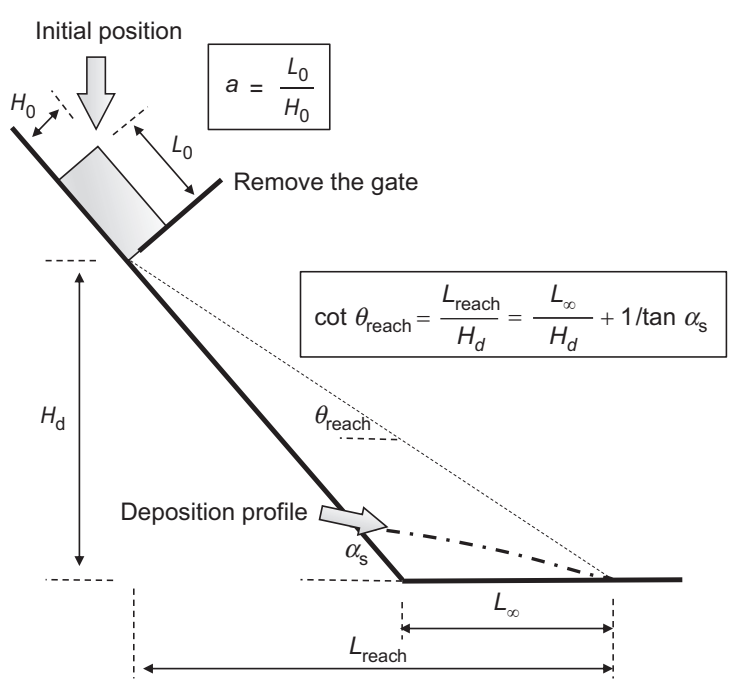

Fig. 9. Experimental set-up of granular flow along a cut slope with a rough sketch of the deposition profile

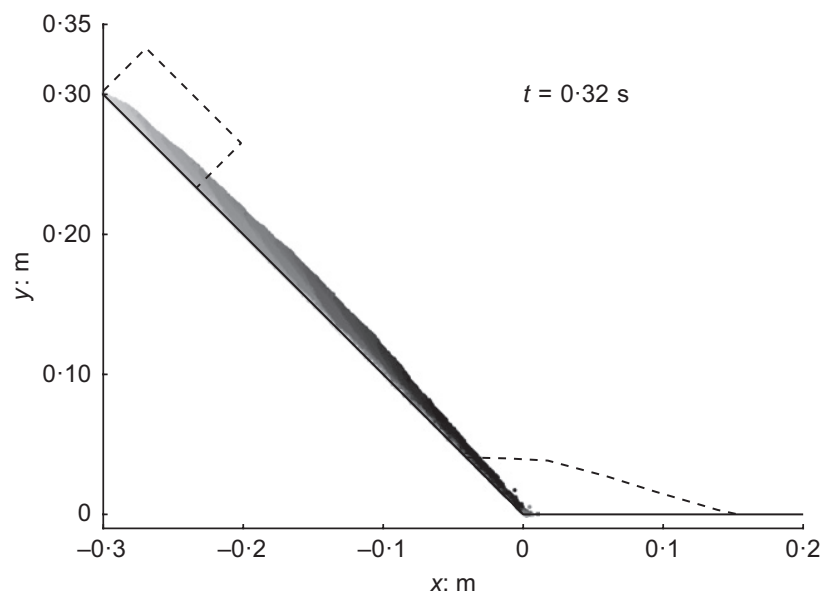

(a)

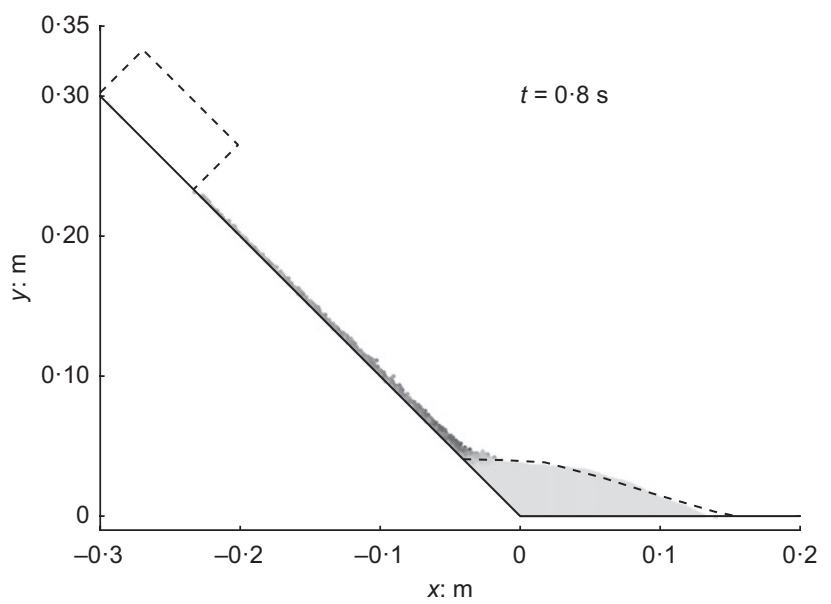

(c) information. Although this relationship could be obtained directly from the power relationship in the literature $\left(L_{\infty} / \sqrt{S_{0}}=L_{\infty} / \sqrt{L_{0} H_{0}}=\left(L_{\infty} / L_{0}\right) \sqrt{\left(L_{0} / H_{0}\right)}=\right.$ $\left.\left(\lambda_{2} a^{\beta}+1\right) / \sqrt{a}, \quad a \geq a_{\min }\right)$, the data in Fig. 8(c) suggest a new scaling law as below.

$$
\frac{L_{\infty}}{\sqrt{S_{\infty}}}=g_{1}\left(a, \phi_{\text {crit }}\right)=\lambda_{3}\left(\phi_{\text {crit }}\right)+\frac{\lambda_{4}\left(\phi_{\text {crit }}\right)}{\sqrt{a}}
$$

The mobility depends on the frictional property of the material. For a given type of granular material, the mobility of granular columns grows with the initial aspect ratio. However, the mobility will reach an asymptotic value when the aspect ratio is very large.

\section{GRANULAR FLOWS ON CUT SLOPES}

Various laboratory experiments have been conducted (Pudasaini et al., 2007; Bryant et al, 2015) in chutes to study dry, granular flows along cut slopes and their deposition at the cut slope toe (Fig. 9), which mimic cut-slope landslides. The granular materials are initially

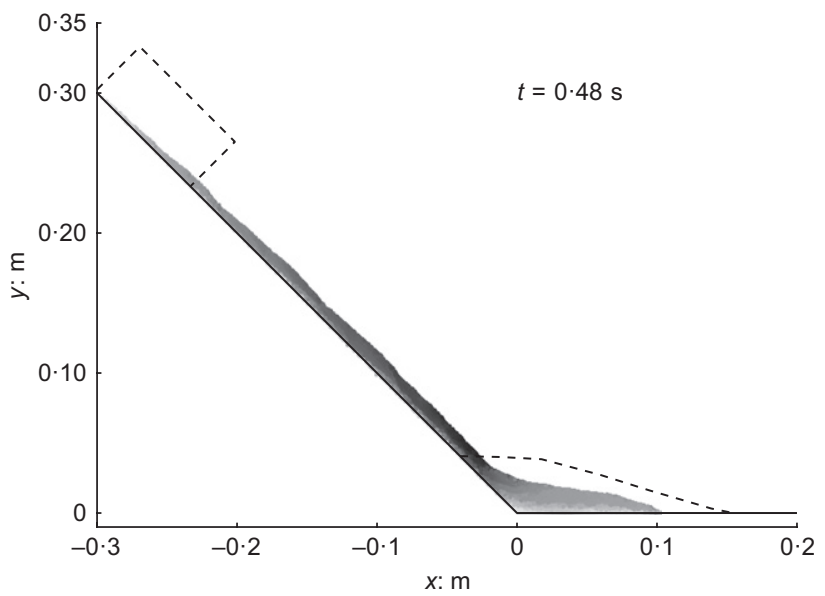

(b)

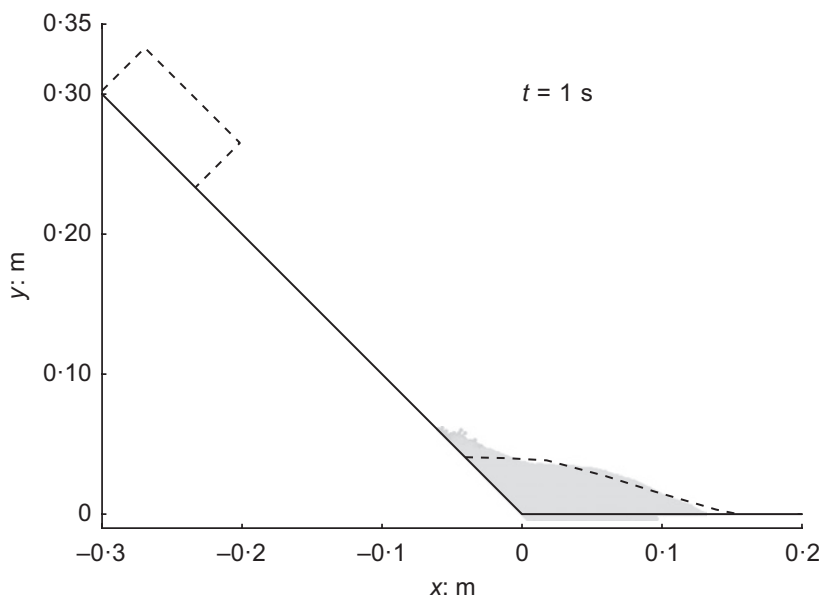

(d)

$|v|: \mathrm{m} / \mathrm{s}$

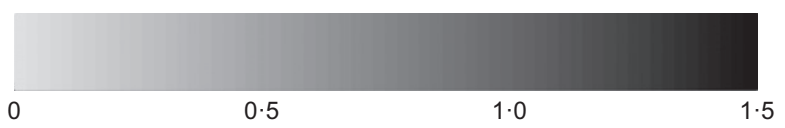

Fig. 10. Velocity field in SPH simulation of granular flow along a $45^{\circ}$ slope, with the initial position and measured deposition profile labelled by the dashed lines (Bryant et al., 2015): (a) $t=0.32 \mathrm{~s}$; (b) $t=0.48 \mathrm{~s}$; (c) $t=0.8 \mathrm{~s}$; (d) $t=1 \mathrm{~s}$ 
contained in a rectangular box on a slope with a slope angle $\alpha_{\mathrm{s}}$ and at a drop height $H_{\mathrm{d}}$. Then, the materials are released by the sudden lifting of a removable gate. The granular materials will flow along the slope and are eventually deposited on a horizontal bed. Experiments of this kind present a challenge for numerical models, as the granular materials impact onto the horizontal beds violently with high velocities, suddenly changing their direction of motion.

\section{Flow simulated by the SPH model}

Bryant et al. (2015) conducted some experiments with sand contained in a box of $L_{0}=96 \mathrm{~mm}$ and $H_{0}=45 \mathrm{~mm}$. The sand is reported to have $\phi_{\text {crit }}=38^{\circ}$ and this value is used in the SPH simulations. The spacing $\Delta l$ is chosen as $0.6 \mathrm{~mm}$ in SPH simulations and the sand mass is discretised into $12075 \mathrm{SPH}$ particles. Two slope angles were used in the experiments. Fig. 10 shows the SPH simulation of the sand flowing down a $45^{\circ}$ slope with a drop height of $233 \mathrm{~mm}$. The slope angle in Fig. 11 is $70^{\circ}$ and the drop height is $385 \mathrm{~mm}$. The initial position and the final deposition of the experiments are indicated with dashed lines. Figs 10(a) and 11(a) show the moment when the sand mass first reaches the horizontal bed. It can be observed that, when the sand mass is released on the $45^{\circ}$ slope, the velocity difference between the front and the rear end is considerable. Therefore, when the front reaches the horizontal bed, the rear end is still stationary and the sand mass is more stretched and thinner compared to the sand mass on the $70^{\circ}$ slope. Figs $10(\mathrm{~b})$ and 10(c) show the deposition process for the flow on the $45^{\circ}$ slope. The front comes to a stop very quickly. Subsequent particles will flow over the stationary layer and then push the front even further. At some time, the run-out will not increase any further and there is still mass left on the slope. This will eventually be deposited at the top of the final heap. Fig. 10(d) compares the final deposition profile between the experiment and the SPH simulation. The model can predict the profile quite well except for some extra mass at the rear end of the

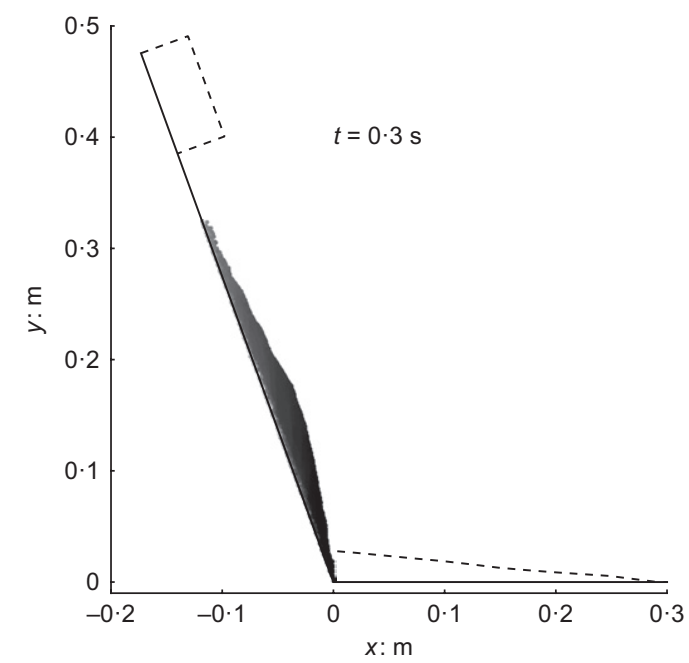

(a)

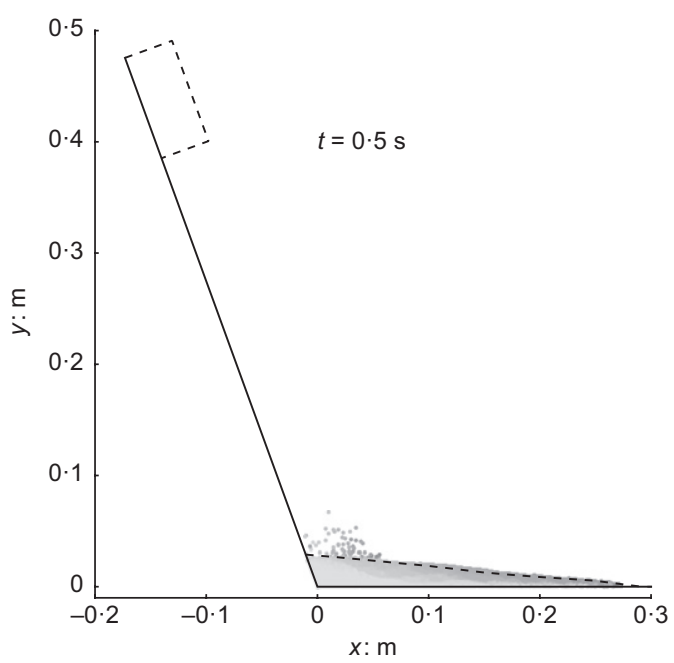

(c)

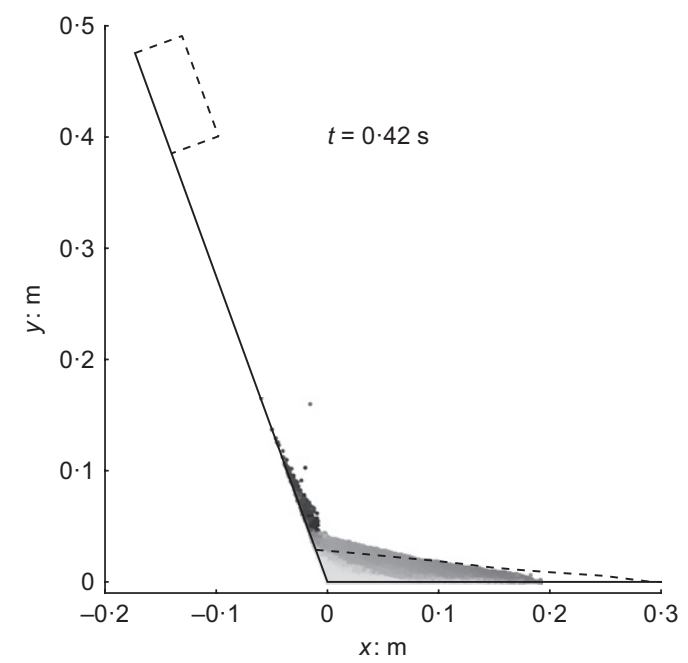

(b)

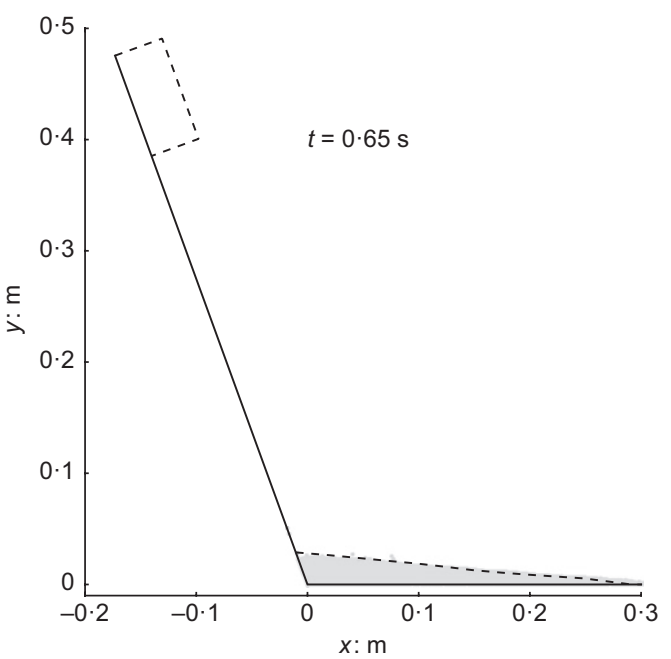

(d)

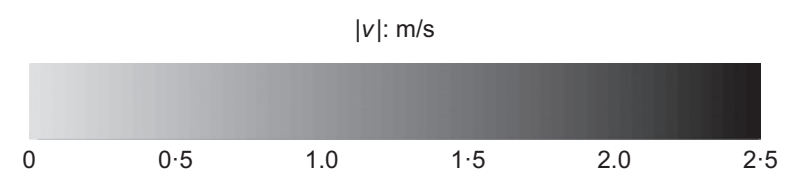

Fig. 11. Velocity field in SPH simulation of granular flow along a $70^{\circ}$ slope, with the initial position and measured deposition profile labelled by the dashed lines (Bryant et al., 2015): (a) $t=0.3 \mathrm{~s}$; (b) $t=0.42 \mathrm{~s}$; (c) $t=0.5 \mathrm{~s}$; (d) $t=0.65 \mathrm{~s}$ 
flow, which is deposited at the top of the heap. This could be attributed to the fact that the width of the box is not exactly equal to the width of the chute in the experiments. The deposition process for the flow on the $70^{\circ}$ slope is illustrated in Figs 11(b) and 11(c). A totally different deposition mechanism is observed. Some front mass is deposited at the toe first. Then, it seems that the rest of the sand mass switches the velocity directions smoothly on top of this stationary layer. Afterwards, the deposition starts with mass near the slope toe and the whole body comes to a stop until the front stops. The predicted final profile also agrees well with the experimental one. In summary, the present model is able to reproduce the flow of dry granular materials down cut slopes and predict the final deposited profiles correctly under various slope conditions.

\section{Verification of the scaling law}

Before moving to the study of landslides involving very complex shapes, the mobility of granular rectangles down a cut slope, and their deposition at the slope toe (Fig. 9), are first studied. It is easy to verify that this problem can be uniquely defined with geometrical parameters $H_{\mathrm{d}}, \alpha_{\mathrm{s}}, H_{0}$, $L_{0}$ and $S_{\infty}$. This rapid flow phenomenon shares some similarity with the aforementioned column collapse phenomenon, because both phenomena involve the acceleration due to gravity, the dissipation of kinetic energy through internal friction and the deposition onto a horizontal bed. Therefore, a similar relationship between the normalised run-out and normalised initial geometrical parameters is expected, that is, $\left(L_{\infty} / \sqrt{S_{\infty}}\right)=g_{2}\left(\alpha_{s}, H_{d} / \sqrt{S_{\infty}}, L_{0} / H_{0}, \phi_{\text {crit }}\right)$. Here, $\alpha_{s}, H_{\mathrm{d}} / \sqrt{S_{\infty}}$ and $L_{0} / H_{0}$ are normalised geometrical parameters, which are expected to play similar roles to the aspect ratio $a$ in equation (10) and their influence on the mobility is investigated. In the subsequent study, the influence of the internal friction angle is not investigated, $\phi_{\text {crit }}$ is fixed as $38^{\circ}$ in all later simulations.

First, simulations are conducted to examine whether the normalised run-out remains the same and the normalised final profiles collapse together for flows of the same normalised geometrical parameters but different crosssectional areas. Various SPH simulations are conducted with the slope angle $\alpha_{\mathrm{s}}$, the normalised drop height $H_{\mathrm{d}} / \sqrt{S_{\infty}}$ and normalised initial shape $L_{0} / H_{0}$ remaining the same as those in the validation examples (Bryant et al., 2015). However, the cross-sectional areas are increased by a factor of 2 or 4 . The normalised final profiles are shown in Fig. 12(a). It is found that the normalised final profiles for the $70^{\circ}$ slope agree exactly with each other. There is some minor discrepancy for the profiles on the $45^{\circ}$ slope. The normalised run-out and the front of the normalised profiles are identical for all test cases. The deposition process on the $45^{\circ}$ slope is characterised by continuous deposition of granular materials on top of the already stationary materials, while the front advances forward at the same time. However, the front will eventually go no further while there is still some sand flowing on the slope which will finally be deposited over the top of the final heap. It seems that, with different cross-sectional areas, the flow and deposition of these materials at the rear end is not properly scaled, which contributes to a minor discrepancy at the top of the heap.

Various SPH simulations are also conducted to investigate the influence of the normalised drop height $H_{\mathrm{d}} / \sqrt{S_{\infty}}$ on the mobility. The normalised final profiles are presented in

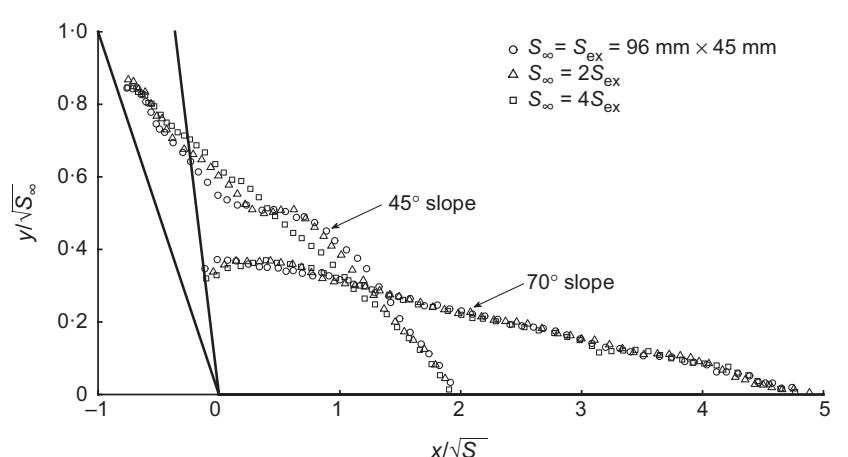

(a)

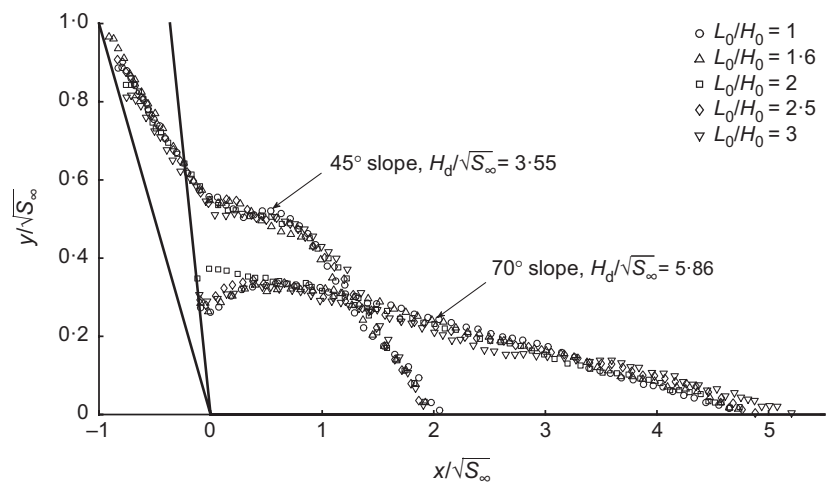

(c)

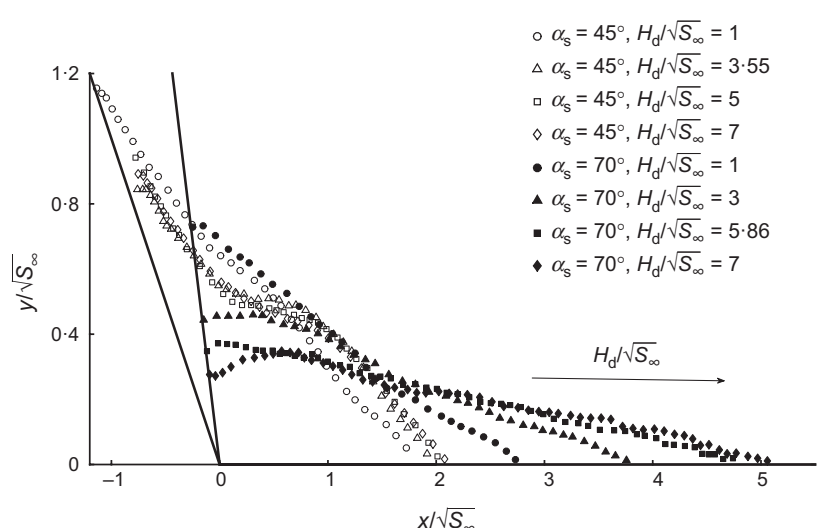

(b)

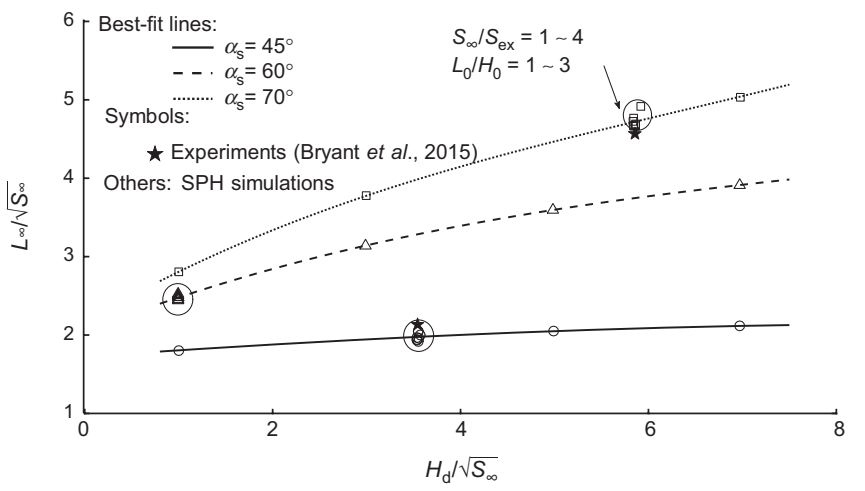

(d)

Fig. 12. Normalised deposition profiles and normalised run-outs of granular rectangles on steep slopes: (a) normalised deposition profiles for granular rectangles with various cross-sectional areas, $S_{\infty}$; (b) normalised deposition profiles for granular rectangles with various normalised drop heights, $H_{\mathrm{d}} / \sqrt{S_{\infty}}$; (c) normalised deposition profiles for granular rectangles with various initial aspect ratios, $\boldsymbol{L}_{0} / H_{0} ;(\mathbf{d})$ relationship between the normalised run-out and the normalised drop height 
Fig. 12(b) with $L_{0} / H_{0}$ fixed at 2 . It can be seen that the mobility grows with the normalised drop height in each slope condition. The growth rate of $L_{\infty} / \sqrt{S_{\infty}}$ with the increase of $H_{\mathrm{d}} / \sqrt{S_{\infty}}$ is not constant, the growth rate decreases and asymptotic $L_{\infty} / \sqrt{S_{\infty}}$ (especially in the $45^{\circ}$ slope simulations) can be observed with large $H_{\mathrm{d}} / \sqrt{S_{\infty}}$.

$L_{0} / H_{0}$ has a limited influence on the mobility, as shown in Fig. 12(c). In these simulations, the slope angle and the normalised drop height are the same and $L_{0} / H_{0}$ varies from 1 to 3 . All deposition profiles are similar, despite some minor discrepancies.

In summary, for this kind of granular flow process, the relationship between the normalised run-out and normalised geometrical parameters is plotted in Fig. 12(d) with both experimental and ISPH results. For tests with various cross-sectional areas and aspect ratios, but with the same slope angle and normalised drop height, the predicted normalised run-out is approximately the same, as indicated by the symbols overlapping together in circles in Fig. 12(d). Therefore, the two main influencing factors are $\alpha_{\mathrm{s}}$ and $H_{\mathrm{d}} / \sqrt{S_{\infty}}$. The normalised drop height has an effect similar to the aspect ratio in the column collapsing process. A greater $H_{\mathrm{d}} / \sqrt{S_{\infty}}$ corresponds to larger mobility. The growth of $L_{\infty} / \sqrt{S_{\infty}}$ with $H_{\mathrm{d}} / \sqrt{S_{\infty}}$ is not linear and the growth rate drops when $H_{\mathrm{d}} / \sqrt{S_{\infty}}$ is large.

In the literature, statistical studies of historical landslide data show that landslides with a greater flowing mass are more mobile and have a smaller reach angle (Corominas, 1996; Legros, 2002). This result is consistent with the relationship obtained from Fig. 12(d). Following the definitions in Fig. 9, the horizontal distance between the head of the landslide source and the distal margin of the deposited mass is $L_{\text {reach }}=L_{\infty}+H_{\mathrm{d}} / \tan \alpha_{\mathrm{s}}$. Then, the extensively used reach angle has the form $\cot \theta_{\text {reach }}=L_{\text {reach }} / H_{\mathrm{d}}=\left(L_{\infty} / H_{\mathrm{d}}\right)+1 / \tan \alpha_{\mathrm{s}}$. The relationship in Fig. 12(d) can be reformulated as $L_{\infty} / \sqrt{S_{\infty}}=\lambda_{5}\left(H_{\mathrm{d}} / \sqrt{S_{\infty}}, \alpha_{\mathrm{s}}\right)\left(H_{\mathrm{d}} / \sqrt{S_{\infty}}\right) . \quad L_{\infty} / \sqrt{S_{\infty}}$ grows with $H_{\mathrm{d}} / \sqrt{S_{\infty}}$ non-linearly and the growth rate becomes smaller for larger $H_{\mathrm{d}} / \sqrt{S_{\infty}}$. Therefore, $\lambda_{5}\left(H_{\mathrm{d}} / \sqrt{S_{\infty}}, \alpha_{\mathrm{s}}\right)$ is a decreasing function with $H_{\mathrm{d}} / \sqrt{S_{\infty}}$. The cotangent of the reach angle can then be expressed as

$$
\cot \theta_{\text {reach }}=\lambda_{5}\left(\frac{H_{\mathrm{d}}}{\sqrt{S_{\infty}}}, \alpha_{\mathrm{s}}\right)+1 / \tan \alpha_{\mathrm{s}}
$$

This equation indicates that the increase of the landslide volume $\left(S_{\infty}\right)$ will result in a decrease of the reach angle, which is exactly the statistical observations of real landslides. Therefore, the relationship obtained from numerical simulations is consistent with observations of historical landslides. This reaffirms the obtained relationship and provides confidence in the further study of landslides involving complex shapes.

\section{IMPLICATIONS OF THE MOBILITY OF \\ CUT-SLOPE LANDSLIDES}

The influence of the source shape, namely, the initial shape of the landslide mass, on the mobility is studied in this section. In the previous study of granular rectangles on cut slopes, the geometry of the source is simple and easy to define, because the base inclines at the same angle as the slope angle and the rectangle is described by an initial length $L_{0}$ and height $H_{0}$. However, most real-world landslides happen with a basal slip surface whose inclination is smaller than the slope angle. For example, Fig. 13 references two historical cut-slope landslides that happened in Hong Kong. Fig. 13(a) concerns the landslide near Victoria Road on 10 August 2002 (GEO, 2004), and Fig. 13(b) shows the landslide near Fei Tsui Road on 13 August 1995 (GEO, 1996). Three types of idealised cut-slope landslides (Figs 13(c)-13(e)) are simulated with the present ISPH model. Attention is focused on the influence of the source shape on the mobility. For type A (Fig. 13(c)), the source is still a rectangle, but the base inclines an angle $\alpha_{\text {sr }}$ with respect to the horizontal. $L_{0} / H_{0}$ of the rectangle varies from 1 to 3. Type B (Fig. 13(d)) mimics landslides similar to the one near Victoria Road. The source is represented by a steep triangle with its base inclined at $\alpha_{\mathrm{sr}}$ to the horizontal, and its back scarp surface inclined at an angle $\alpha_{\text {back }}$. Type C (Fig. 13(e)) is an abstraction of the landslide near Fei Tsui Road. The back scarp surface intersects the horizontal top of the cut slope. Simulations with $\alpha_{\mathrm{sr}}=35^{\circ}$ or $45^{\circ}$ are conducted on $60^{\circ}$ and $70^{\circ}$ cut slopes. The internal friction angle is selected as $38^{\circ}$, so that the source mass is not stable in the first instance. The choosing of a strength parameter which leads the initial mass to be unstable enables the simplified simulation of a triggering process, which could be caused by various physical processes such as the loss or decrease of suction after heavy rains, the removal of vegetation, or an earthquake.

One overall observation is that, with the same slope angle and normalised drop height, the normalised run-out for these idealised cut-slope landslides is smaller than that of corresponding granular rectangles. Several deposition profiles on $70^{\circ}$ slopes with a fixed $H_{\mathrm{d}} / \sqrt{S_{\infty}}$ of 5.86 are plotted in Fig. 14. It is observed that the geometrical parameters of the source shape play an important role in the mobility of landslides.

An interesting feature of an initial discontinuity in the landslide mass is observed in these simulations. Fig. 15 shows the velocity of an idealised landslide mass a very short period $(0.045 \mathrm{~s})$ after the slope loses stability. The mass above the initial discontinuity plane gains momentum quickly and forms the front of the flow. The material below the initial discontinuity plane is relative slow. It will follow and form the rear end of the flow. This discontinuity phenomenon is also observed in the collapse experiments of granular columns. At the start of the collapse, only grains above an initial failure plane will gain momentum and grains below this initial failure plane remain static. The initial failure plane inclines at $(\pi / 4)+\left(\phi_{\text {crit }} / 2\right)$ to the horizontal for granular columns. In the simulations of idealised landslides, the initial discontinuity plane is not a straight line but is curved. Its inclination (approximately $\theta_{\mathrm{id}}=55^{\circ}$ in the present simulations) is also slightly smaller than $(\pi / 4)+\left(\phi_{\text {crit }} / 2\right)=64^{\circ}$. In addition, a general trend is observed in all simulations of idealised landslides that a greater area above the initial discontinuity plane $\left(S_{\text {front }}\right.$ in Fig. 15) leads to more mass at the front and a greater normalised run-out. Here, $S_{\text {front }}$ is approximately estimated to be the area above a straight line inclining at $\theta_{\text {id }}=55^{\circ}$, as shown in Fig. 15. Fig. 16 plots the relationship between the normalised run-out and the proportion of front mass to the whole mass $\left(S_{\text {front }} / S_{\infty}\right)$. Two slope conditions are presented. The first one refers to idealised landslides on a $70^{\circ}$ cut slope with $H_{\mathrm{d}} / \sqrt{S_{\infty}}$ of $5 \cdot 86$, and the second one is on a $60^{\circ}$ cut slope with $H_{\mathrm{d}} / \sqrt{S_{\infty}}$ of 1 . The normalised run-outs of granular rectangles are represented with open diamonds. In comparison, all idealised landslides have smaller normalised run-out when the slope angle and normalised drop height are the same. In addition, for each slope condition, the SPH results seem to collapse onto one unique curve. Therefore, of the factors depicting the source shape of landslides, the ratio $S_{\text {front }} / S_{\infty}$ is the dominant one in determining the run-out. In summary, the present study shows that the normalised run-out of cut-slope landslides is mainly determined by the slope angle $\alpha_{\mathrm{s}}$, the drop height $H_{\mathrm{d}} / \sqrt{S_{\infty}}$ and a dimensionless parameter $S_{\text {front }} / S_{\infty}$. 

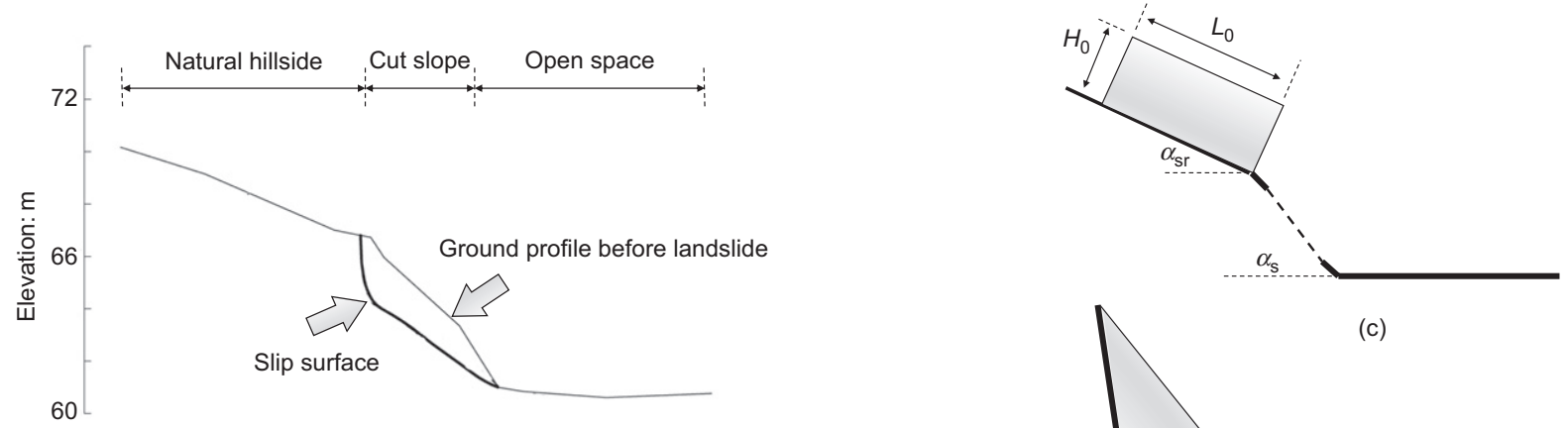

(a)
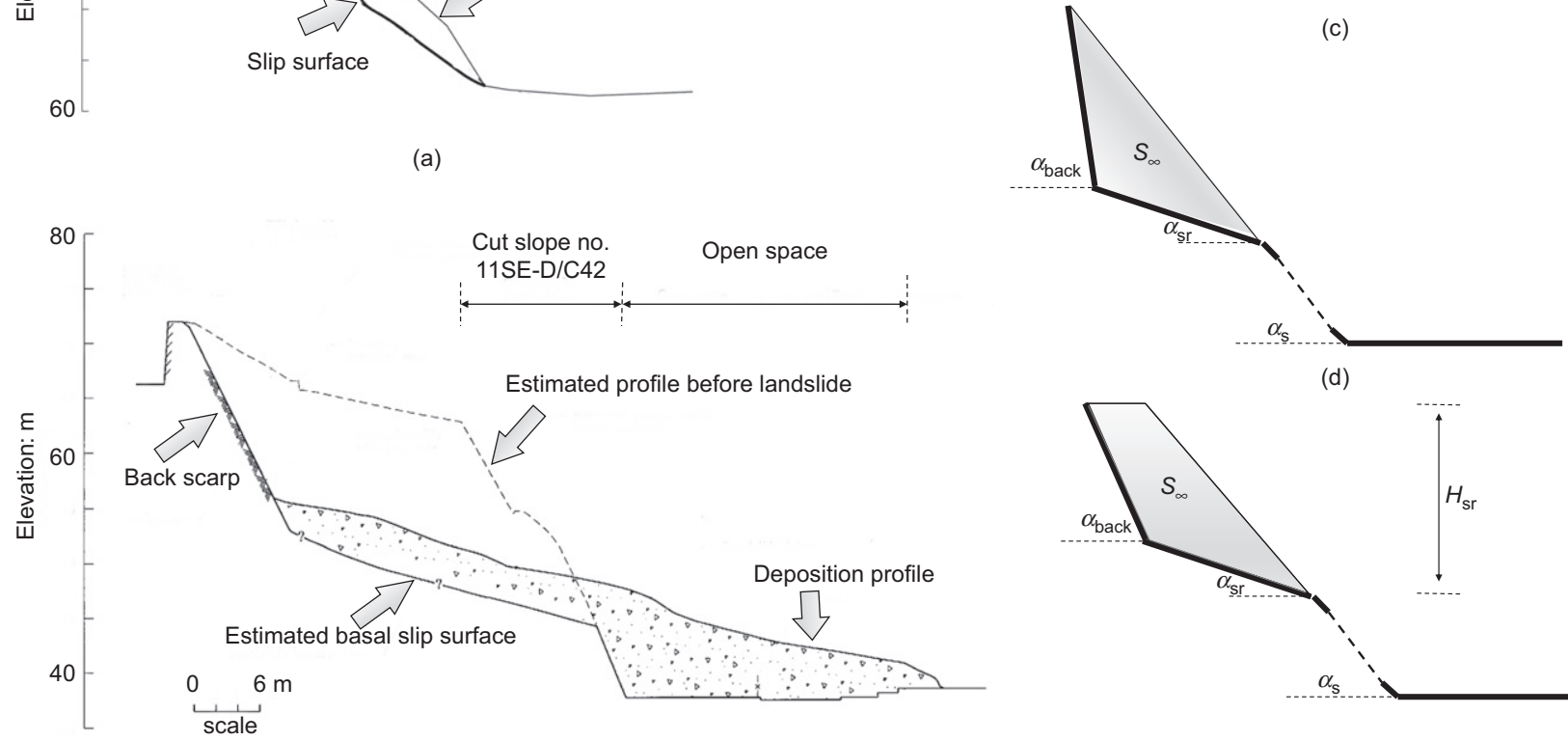

(d)

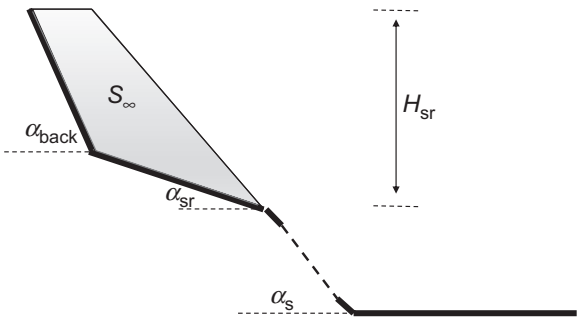

(b)

(e)

Fig. 13. Historical landslides and three idealised cut-slope landslides simulated with the SPH model: (a) a cut-slope landslide near Victoria Road, Hong Kong on 10 August 2002; (b) a cut-slope landslide near Fei Tsui Road, Hong Kong on 13 August 1995; (c) type A idealised landslide; (d) type $B$ idealised landslide; (e) type $\mathrm{C}$ idealised landslide

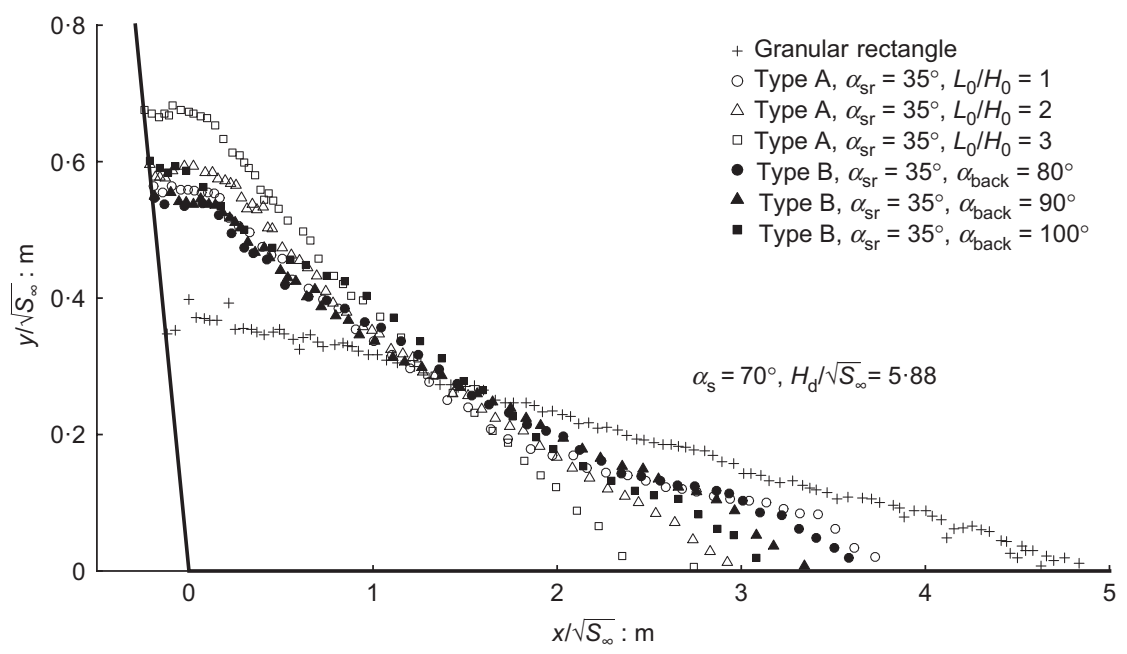

Fig. 14. Normalised deposition profiles for various idealised cut-slope landslides

\section{CONCLUSIONS}

This paper presents a study using ISPH simulations of granular materials flowing down cut slopes and depositing at the slope toe. In the ISPH model, the granular materials are modelled as a rigid and perfectly plastic material with a Coulomb yield surface. Dilatancy is ignored and thus the material is assumed to be incompressible. The coupled continuity equation and momentum equations are solved using a semi-implicit algorithm.

The present ISPH model is validated and its results are carefully compared with various controlled experiments. The
ISPH model correctly predicts the heap formed by slowly poured granular materials, and the results indicate that the heap angle and the internal friction angle follow the relationship $\tan \mathrm{AOR} \approx \sin \phi_{\text {crit }}-$ a hypothesis on slip lines of zero extension proposed by Roscoe (1970). The model is also verified against column collapse experiments. All the key features can be successfully reproduced, and the capability of predicting the final profiles in various configurations and with various granular materials is extensively verified. Then, the model is used to simulate the release of granular rectangles on cut slopes to investigate the deposit 


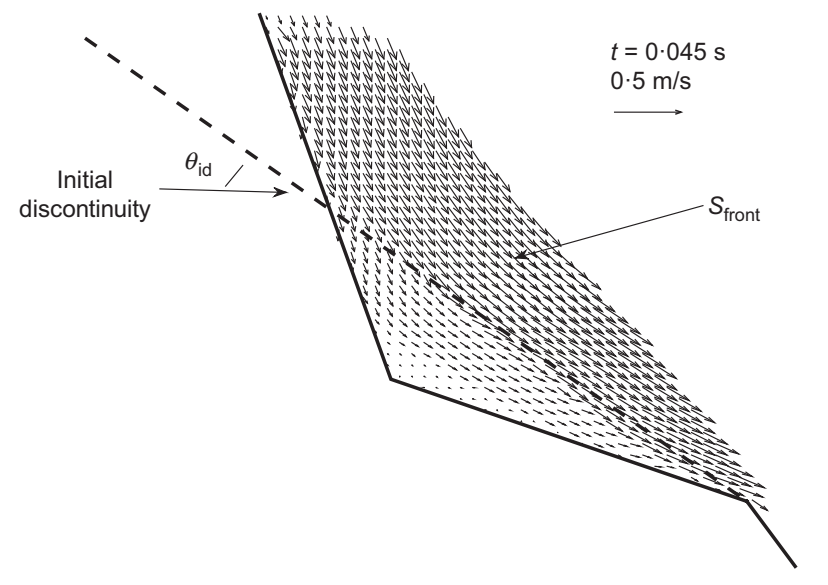

Fig. 15. SPH-predicted velocity field of the landslide mass $0 \cdot 045 \mathrm{~s}$ after the initiation. $\left(S_{\infty}=0.0043 \mathrm{~m}^{2}, \alpha_{\mathrm{s}}=70^{\circ}, \alpha_{\mathrm{sr}}=35^{\circ}, \alpha_{\text {back }}=80^{\circ}\right.$, $\left.H_{\mathrm{sr}}=0 \cdot 15 \mathrm{~m}\right)$

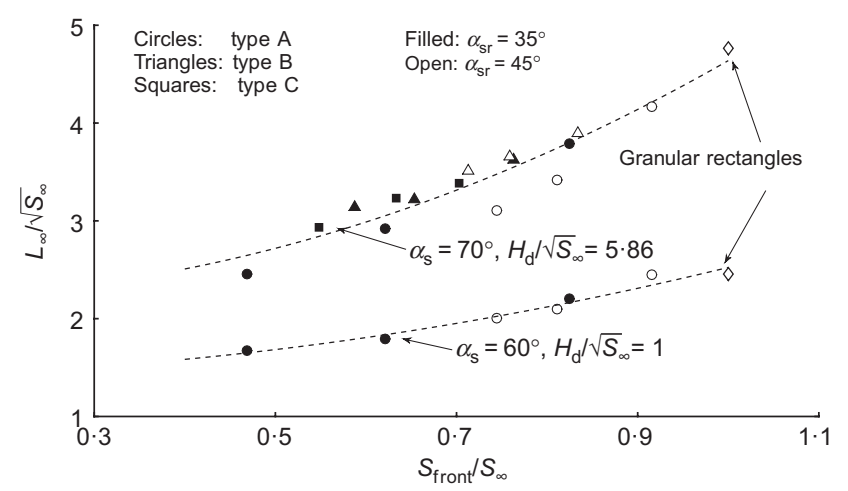

Fig. 16. Relationships between the normalised run-out and the ratio of the front mass to the whole mass in three types of idealised cut-slope landslides

morphologies. It is observed that the mathematical model reproduces the flow and predicts the final deposition profiles correctly under various conditions.

A main contribution of this study is to have used the simulation results to study the run-out and mobility of granular columns, granular rectangles on steep slopes and idealised cut-slope landslides. To better describe the mobility of granular columns, a new normalised run-out is proposed, which is also supported by experimental results, DEM simulations and results from continuum-based simulations. Additionally, a normalised relationship for the run-out of granular rectangles on cut slopes is also proposed. The normalised run-out $L_{\infty} / \sqrt{S_{\infty}}$ is found to be affected mainly by the slope angle $\alpha_{\mathrm{s}}$ and the normalised drop height $H_{\mathrm{d}} / \sqrt{S_{\infty}}$, with $L_{\infty} / \sqrt{S_{\infty}}$ increasing with $H_{\mathrm{d}} / \sqrt{S_{\infty}}$ non-linearly.

Three types of idealised cut-slope landslides are simulated with the aim of studying the influence of the source shape on mobility. It is found that the normalised run-out of these idealised cut-slope landslides is smaller than that of granular rectangles with the same slope angle and normalised drop height. The difference between the run-outs is found to be dominantly determined by the ratio of the mass above an initial discontinuity plane to the whole mass.

\section{ACKNOWLEDGEMENTS}

The research is supported by the European Union Seventh Framework Program (FP7/2007-2013) under grant agreement no. PIAG-GA-2012-324522 'MPM-DREDGE'. Dr He would also thank the Raymond and Helen Kwok Scholarship from the Cambridge Trust and Jesus College.

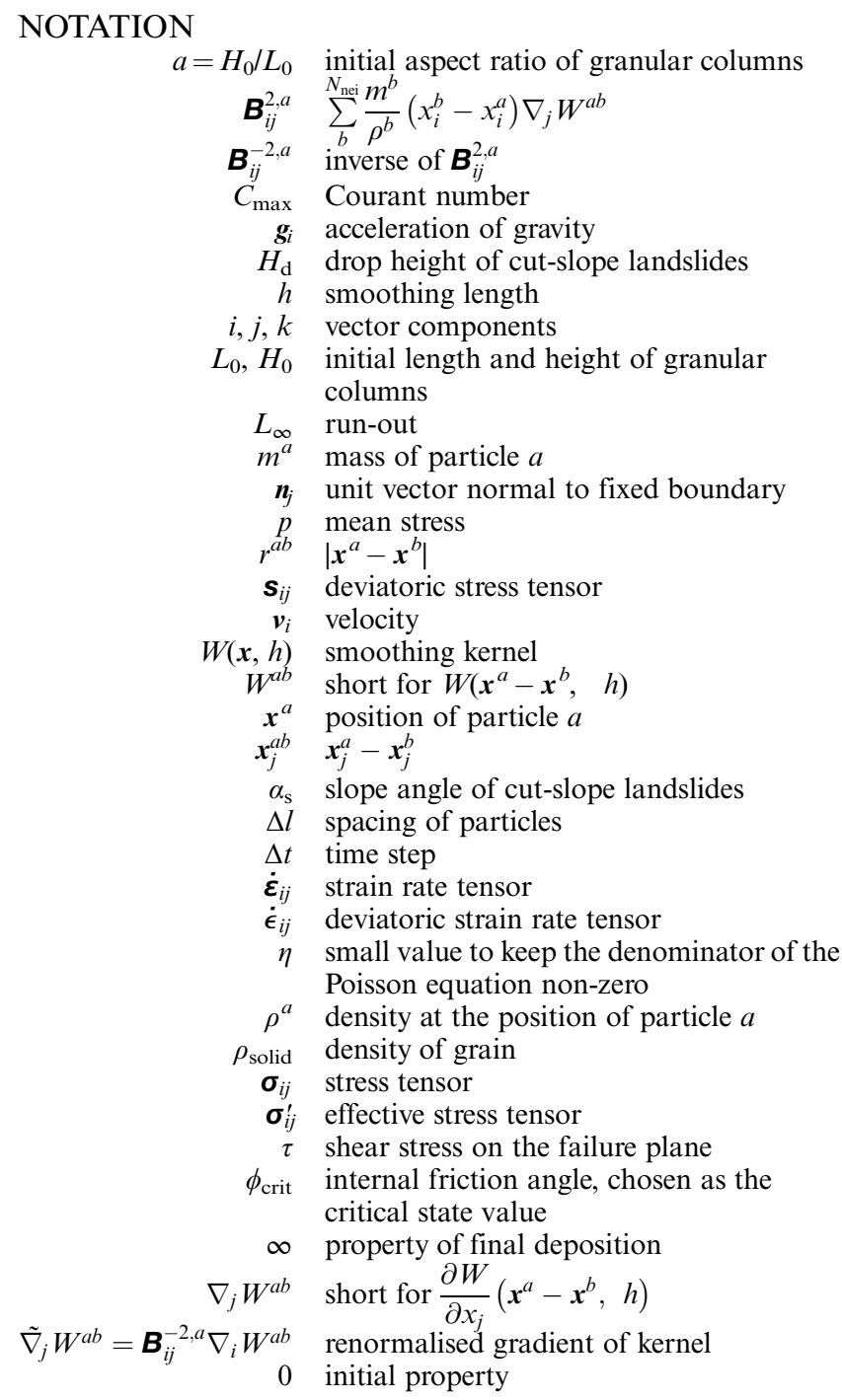

\section{REFERENCES}

Ai, J., Langston, P. A. \& Yu, H. S. (2014). Discrete element modelling of material non-coaxiality in simple shear flows. Int. J. Numer. Analyt. Methods Geomech. 38, No. 6, 615-635.

An, Y., Wu, Q., Shi, C. \& Liu, Q. (2016). Three-dimensional smoothed-particle hydrodynamics simulation of deformation characteristics in slope failure. Géotechnique 66, No. 8, 670-680, http://dx.doi.org/10.1680/jgeot.15.P.222.

Balmforth, N. J. \& Kerswell, R. R. (2005). Granular collapse in two dimensions. J. Fluid Mech. 538, No. 1, 399-428.

Bolton, M. D. (1986). The strength and dilatancy of sands. Géotechnique 36, No. 1, 65-78, http://dx.doi.org/10.1680/geot. 1986.36.1.65.

Bryant, S. K., Take, W. A., Bowman, E. T. \& Millen, M. D. L. (2015). Physical and numerical modelling of dry granular flows under Coriolis conditions. Géotechnique 65, No. 3, 188-200, http://dx.doi.org/10.1680/geot.13.P.208.

Bui, H. H., Fukagawa, R., Sako, K. \& Ohno, S. (2008). Lagrangian meshfree particles method (SPH) for large deformation and failure flows of geomaterial using elastic-plastic soil constitutive model. Int. J. Numer. Analyt. Methods Geomech. 32, No. 12, $1537-1570$.

Bui, H. H., Fukagawa, R., Sako, K. \& Wells, J. C. (2011). Slope stability analysis and discontinuous slope failure simulation by elasto-plastic smoothed particle hydrodynamics (SPH). 
Géotechnique 61, No. 7, 565-574, http://dx.doi.org/10.1680/ geot.9.P.046.

Corominas, J. (1996). The angle of reach as a mobility index for small and large landslides. Can. Geotech. J. 33, No. 2, 260-271.

Crosta, G. B., Imposimato, S. \& Roddeman, D. (2009). Numerical modeling of 2-D granular step collapse on erodible and nonerodible surface. J. Geophys. Res.: Solid Earth 114, No. F3, F03020.

Forterre, Y. \& Pouliquen, O (2008). Flows of dense granular media. Ann. Rev. Fluid Mech. 40, No. 1, 1-24.

GDR MiDi group (2004). On dense granular flows. Eur. Phys. J. E, Soft Matter 14, No. 4, 341-365.

GEO (Geotechnical Engineering Office) (1996). Report on the Fei Tsui road landslide of 13 August 1995. Hong Kong: Hong Kong Government.

GEO (2004). Detailed study of the 10 August 2002 landslide incident on a cut slope at Victoria Road, Hong Kong. Hong Kong, PR China: Hong Kong Government.

He, X (2016) SPH study of flow-type landslides and their interaction with rigid barriers. PhD thesis, University of Cambridge, Cambridge, UK.

He, X. \& Liang, D. (2015). Study of the runout of granular columns with SPH methods. Int. J. Offshore Polar Engng 25, No. 4, 281-287.

Hunter, G. \& Fell, R. (2003). Travel distance angle for 'rapid' landslides in constructed and natural soil slopes. Can. Geotech. J. 40, No. 6, 1123-1141.

Ionescu, I. R., Mangeney, A., Bouchut, F. \& Roche, O. (2015). Viscoplastic modeling of granular column collapse with pressure-dependent rheology. J. Non-Newtonian Fluid Mech. 219, 1-18.

Iverson, R. M. \& Denlinger, R. P. (2001). Flow of variably fluidized granular masses across three-dimensional terrain: 2. Numerical predictions and experimental tests. J. Geophys. Res. 106, No. B1, $553-552$.

Lacaze, L. \& Kerswell, R. (2009). Axisymmetric granular collapse: a transient 3D flow test of viscoplasticity. Phys. Rev. Lett. 102, No. 10, 108305.

Lacaze, L., Phillips, J. C. \& Kerswell, R. R. (2008). Planar collapse of a granular column: experiments and discrete element simulations. Phys. Fluids 20, No. 6, 063302.

Lajeunesse, E., Monnier, J. B. \& Homsy, G. M. (2005). Granular slumping on a horizontal surface. Phys. Fluids 17, No. 10, 103302.

Lee, E. S., Moulinec, C., Xu, R., Violeau, D., Laurence, D. \& Stansby, P. (2008). Comparisons of weakly compressible and truly incompressible algorithms for the SPH mesh free particle method. J. Comput. Phys. 227, No. 18, 8417-8436.

Legros, F. (2002). The mobility of long-runout landslides. Engng Geol. 63, No. 3-4, 301-331.

Liang, D. (2010). Evaluating shallow water assumptions in dam-break flows. Proc. Instn Civ. Engrs - Water Manage. 163 No. 5, 227-237.

Liang, D. \& He, X. (2014). A comparison of conventional and shear-rate dependent Mohr-Coulomb models for simulating landslides. J. Mount. Sci. 11, No. 6, 1478-1490.

Lube, G., Huppert, H. E., Sparks, R. S. J. \& Freundt, A. (2005). Collapses of two-dimensional granular columns. Phys. Rev. E-Statist., Nonlinear, Soft Matter Phys. 72, No. 4, 041301.

Lube, G., Huppert, H. E., Sparks, R. S. J. \& Freundt, A. (2011). Granular column collapses down rough, inclined channels. J. Fluid Mech. 675, 347-368.

Monaghan, J. J. (1992). Smoothed particle hydrodynamics. Ann. Rev. Astronomy Astrophys. 30, 543-574.

Nguyen, C. T., Nguyen, C. T., Bui, H. H., Nguyen, G. D. \& Fukagawa, R. (2016). A new SPH-based approach to simulation of granular flows using viscous damping and stress regularisation. Landslides 14, No. 1, 69-81.

Pudasaini, S. P., Hutter, K., Hsiau, S. S., Tai, S. C., Wang, Y. \& Katzenbach, R. (2007). Rapid flow of dry granular materials down inclined chutes impinging on rigid walls. Phys. Fluids 19, No. 5, 053302.

Randles, P. W. \& Libersky, L. D. (1996). Smoothed particle hydrodynamics: some recent improvements and applications. Comput. Methods Appl. Mech. Engng 139, No. 1-4, 375-408

Roscoe, K. H. (1970) The influence of strains in soil mechanics. Géotechnique 20, No. 2, 129-170, http://dx.doi.org/10.1680/ geot.1970.20.2.129.

Savage, S. B. \& Hutter, K. (1989). The motion of a finite mass of granular material down a rough incline. J. Fluid Mech. 199, $177-215$.

Schofield, A. N. \& Wroth, C. P. (1968). Critical state soil mechanics. London, UK: McGraw-Hill.

Shao, S. (2012). Incompressible smoothed particle hydrodynamics simulation of multifluid flows. Int. J. Numer. Methods Fluids 69, No. 11, 1715-1735.

Staron, L. \& Hinch, E. J. (2005). Study of the collapse of granular columns using DEM numerical simulation. J. Fluid Mech. $\mathbf{5 4 5}$ 0501022.

Utili, S., Zhao, T. \& Houlsby, G. T. (2015). 3D DEM investigation of granular column collapse: evaluation of debris motion and its destructive power. Engng Geol. 186, 3-16. 